\title{
Dark Matter constraints on composite Higgs models
}

\author{
Nayara Fonseca, ${ }^{a, b}$ Renata Zukanovich Funchal, ${ }^{a}$ Andre Lessa ${ }^{a}$ \\ and Laura Lopez-Honorez ${ }^{c}$ \\ ${ }^{a}$ Instituto de Física, Universidade de São Paulo, \\ R. do Matão 187, São Paulo, SP 05508-900, Brazil \\ ${ }^{b}$ Center for Theoretical Physics, Massachusetts Institute of Technology, \\ Cambridge, MA 02139, U.S.A. \\ ${ }^{c}$ Vrije Universiteit Brussel and The International Solvay Institutes, \\ Pleinlaan 2, B-1050 Brussels, Belgium \\ E-mail: nayara@if.usp.br, zukanov@if.usp.br, lessa@if.usp.br, \\ llopezho@vub.ac.be
}

AbSTRACT: In composite Higgs models the pseudo-Nambu-Goldstone Boson (pNGB) nature of the Higgs field is an interesting alternative for explaining the smallness of the electroweak scale with respect to the beyond the Standard Model scale. In non-minimal models additional pNGB states are present and can be a Dark Matter (DM) candidate, if there is an approximate symmetry suppressing their decay. Here we assume that the low energy effective theory (for scales much below the compositeness scale) corresponds to the Standard Model with a pNGB Higgs doublet and a pNGB DM multiplet. We derive general effective DM Lagrangians for several possible DM representations (under the SM gauge group), including the singlet, doublet and triplet cases. Within this framework we discuss how the DM observables (relic abundance, direct and indirect detection) constrain the dimension- 6 operators induced by the strong sector assuming that DM behaves as a Weakly Interacting Particle (WIMP) and that the relic abundance is settled through the freeze-out mechanism. We also apply our general results to two specific cosets: $\mathrm{SO}(6) / \mathrm{SO}(5)$ and $\mathrm{SO}(6) / \mathrm{SO}(4) \times \mathrm{SO}(2)$, which contain a singlet and doublet DM candidate, respectively. In particular we show that if compositeness is a solution to the little hierarchy problem, representations larger than the triplet are strongly disfavored. Furthermore, we find that composite models can have viable DM candidates with much smaller direct detection cross-sections than their non-composite counterparts, making DM detection much more challenging.

Keywords: Technicolor and Composite Models, Phenomenological Models

ArXiv EPrint: 1501.05957 


\section{Contents}

1 Introduction 1

2 General composite Dark Matter 3

2.1 Aspects of the effective Dark Matter Lagrangian 5

$\begin{array}{lll}2.2 & \text { The effect of higher dimensional operators } & 7\end{array}$

3 Results $\quad 10$

$\begin{array}{lll}3.1 & \text { Singlet DM } & 11\end{array}$

3.1.1 The generic composite singlet DM 11

3.1.2 Scan results 12

$\begin{array}{ll}\text { 3.1.3 Experimental constraints } & 14\end{array}$

$\begin{array}{ll}\text { 3.1.4 Specific case: } \mathcal{G} / \mathcal{H}=\mathrm{SO}(6) / \mathrm{SO}(5) & 16\end{array}$

$\begin{array}{lll}3.2 & \text { Doublet DM } & 17\end{array}$

$\begin{array}{lll}3.2 .1 & \text { Generic composite doublet DM } & 18\end{array}$

$\begin{array}{lll}3.2 .2 & \text { Scan results and experimental constraints } & 20\end{array}$

3.2.3 Specific case: $\mathcal{G} / \mathcal{H}=\mathrm{SO}(6) / \mathrm{SO}(4) \times \mathrm{SO}(2)$

3.3 Triplet DM and higher representations 24

3.3.1 Generic composite triplet DM 25

$\begin{array}{lll}3.3 .2 & \text { Scan results and experimental constraints } & 26\end{array}$

$\begin{array}{lll}3.3 .3 & \text { Higher representations } & 29\end{array}$

$\begin{array}{llr}4 & \text { Conclusions } & 29\end{array}$

A Dimension six operators for composite DM 31

A.1 Singlet DM 32

A.2 Doublet Dark Matter 33

A.3 Real representations $\quad 35$

\section{Introduction}

Despite being an enormous triumph to the Standard Model (SM), the discovery of the Higgs boson [1-5] has established the question of how the electroweak scale is stabilized under the large corrections from new physics at ultra-violet (UV) scales. The quadratic sensitivity of the Higgs mass to UV physics, also known as the hierarchy problem, has been one of the leading motivations for searches of new physics at the LHC. One of the most investigated solutions to the hierarchy problem is Supersymmetry. Besides providing a mechanism for stabilizing the electroweak (EW) scale, the Minimal Supersymmetric Standard Model (for reviews see [6-8]) or MSSM also has several attractive features, such as gauge coupling 
unification and viable dark matter candidates. However, current LHC searches and the measured Higgs mass impose severe constraints on the MSSM.

Composite Higgs models [9-14] are also an interesting solution to the hierarchy problem. Unlike the MSSM, where the Higgs boson is a fundamental scalar, in composite models the Higgs doublet is a pseudo-Nambu-Goldstone Boson (pNGB) appearing in the low energy theory as a result of the spontaneous breaking of a global symmetry $(\mathcal{G} \rightarrow \mathcal{H})$ by a new strong sector dynamics. In analogy to the pions in QCD, the Higgs doublet only has derivative couplings and is exactly massless, except for corrections due to the explicit breaking of $\mathcal{G}$. In the Minimal Composite Higgs Model (MCHM) [15], based on the coset $\mathrm{SO}(5) / \mathrm{SO}(4)$, the four pNGBs correspond to the complex Higgs doublet. Nonetheless, other cosets are possible and may contain a higher number of pNGBs degrees-of-freedom. It is then an interesting question whether one of the additional pseudo Nambu-Goldstone bosons may explain the observed dark matter component of the universe. Since this requires at least five pNGBs (the Higgs doublet plus one DM state), one must consider extensions of the MCHM. The simplest scenario corresponds to the $\mathrm{SO}(6) / \mathrm{SO}(5)$ coset $[16,17]$, which provides exactly one additional pNGB. For a model based on the coset $\mathrm{SO}(7) / G 2$, see e.g. [18].

Possible explanations to the DM question can now be tested by an impressive number of experiments, possibly bringing us at the edge of discovery. Four complementary approaches are followed to identify DM as a new WIMP. Direct searches are looking for DM scattering off heavy nuclei in underground detectors. For the mass ranges that we are interested in, LUX is for the time being the most sensitive experiment [19], reaching scattering cross-sections of the order of $10^{-45} \mathrm{~cm}^{2}$ (for masses around $30 \mathrm{GeV}$ ). In this field, the next data releases are expected for 2015 (LUX-1 year data) increasing the sensitivity by a factor 5. From 2018, the next generation of experiments with multi-tonne Xenon (Xenon1T, LUX-Zeplin) should reach cross-sections down to $10^{-47} \mathrm{~cm}^{2}$. Indirect searches aim at detecting the products of DM annihilation or decay in the form of gamma-rays, neutrinos or antimatter cosmic rays. The Fermi-LAT telescope, which is studying the gamma-ray flux from multiple astrophysical sources, has provided the first strong exclusion limits on DM annihilation cross-sections excluding $\left\langle\sigma_{\text {ann }} . v\right\rangle \sim 3 \times 10^{-26} \mathrm{~cm}^{3} / \mathrm{s}$ for candidates with masses up to $\sim 100 \mathrm{GeV}$ for a $100 \%$ annihilation into $\bar{b} b[20,21]$. It is very likely that new data will soon be available with e.g. AMS-02 for antimatter cosmic rays, Ice-Cube for neutrinos, HESS-II for gamma-rays and with a new generation of experiments, such as Gamma-400 and the CTA. From 2018 onwards, see e.g. [22] for a review, the new generation of indirect detection experiments will improve considerably the current results. Collider searches can also constrain DM scenarios through missing energy searches, although in most cases the constraints are strongly model dependent. In addition, if dark matter has non-negligible couplings to the Higgs field, the current measurement of the Higgs decays can also be relevant for testing DM scenarios [23-25]. Finally, astro-cosmo probes can provide complementary constraints, testing e.g. distortions of the Cosmic Microwave Background (CMB) signal due to energy injection from DM annihilation in the early universe, see e.g. [26-29].

All the data provided by the complementary searches listed above strongly constrains dark matter models. In particular, the composite Higgs model $\mathrm{SO}(6) / \mathrm{SO}(5)$ can only satisfy all the current DM and LHC constraints if the compositeness scale $(F)$ is $\gtrsim 1 \mathrm{TeV}$ 
and if $m_{\mathrm{DM}} \gtrsim 200 \mathrm{GeV}$, as discussed in detail in refs. [16, 17]. Due to the large amount of data available from direct, indirect and LHC searches, it is possible to formulate even more general statements on the possible composite scenarios, if these are required to provide a DM candidate behaving as a WIMP and accounting for all the DM abundance. This is the main purpose of this work. Here we consider the class of composite models where the only composite states present in the low energy effective theory are the Higgs doublet and the DM multiplet, and both are pNGBs. The possible strong resonances and remaining pNGBs are assumed to be decoupled in the effective Lagrangian, either because they are significantly heavier than the Higgs and the DM multiplet or because they correspond to gauge degrees of freedom, due to a partly gauged $\mathcal{G}$. We discuss this possibility with a minimal knowledge of the UV completion. In particular, we do not specify the fermion representations under $\mathcal{G}$, but parametrize their effects instead. Within this framework, any composite Higgs model containing a DM candidate can be mapped at low energies ( $\ll \simeq 4 \pi F)$ to an effective Lagrangian containing the Standard Model (including the Higgs doublet) and the DM multiplet.

In section 2 we define the class of models we will consider and present the basic framework used to compute our results. In section 3 we classify the different scenarios according to the DM representation under $\mathrm{SO}(4)$ and discuss each case separately. The simplest case, consisting of a singlet DM, is discussed in detail in section 3.1. A minimal realization of this scenario can be obtained for $\mathcal{G} / \mathcal{H}=\mathrm{SO}(6) / \mathrm{SO}(5)$, as shown in refs. [16, 17]. The next simplest scenario consistent with $\mathrm{SO}(4) \subset \mathcal{H}$ is the complex doublet $\mathrm{DM}$ case, discussed in section 3.2. Although we once again present our results in a model independent way, we also discuss a realization of the doublet DM case, corresponding to the $\mathrm{SO}(6) / \mathrm{SO}(4) \times \mathrm{SO}(2)$ coset. In section 3.3 we present the results for the triplet and general constraints on higher representations. Finally, in section 4 we summarize our results and present the conclusions. In the appendix A we define our notation and discuss in detail the derivation of the effective Lagrangians used in our calculations.

\section{General composite Dark Matter}

In composite Higgs models [30-36], the strong interactions responsible for compositeness are assumed to break a global symmetry group $\mathcal{G}$ down to a smaller symmetry group $\mathcal{H}$ at the $F$ scale. The low energy spectrum, at energies $\ll \Lambda \simeq 4 \pi F$, consists of the massless Nambu-Goldstone Boson (NGB) modes with an effective Lagrangian completely fixed by the symmetry breaking pattern $\mathcal{G} \rightarrow \mathcal{H}$. For a general $\mathcal{G} / \mathcal{H}$ breaking, the effective interactions of the Nambu-Goldstone modes to lowest order in $\mathcal{O}(1 / F)$ are given by the Callan, Coleman, Wess, Zumino (CCWZ) Lagrangian [37, 38]. In order to avoid large corrections to EW precision observables, we assume $\mathrm{SO}(4) \subset \mathcal{H}$, which implies that the CCWZ Lagrangian considered here is $\mathrm{SO}(4)$ invariant.

If one of the NGBs must play the role of the Higgs doublet, $\mathcal{G}$ must be explicitly broken, resulting in a non-flat potential for the pseudo-Goldstone fields. Here we assume the partial compositeness scenario, where the explicit breaking is restricted to Yukawa and gauge interactions between the SM and the strong sector. Once these interactions 
are determined, the effective scalar potential for the pNGBs can be computed up to form factors describing the strong dynamics. Since the effective scalar potential is generated only at loop level, the pNGBs are expected to have masses $\lesssim F$. The fine-tuning in the Higgs potential is given by the separation between the $F$ and EW scales. A fine-tuning not worse than $1 \%$ implies:

$$
F \lesssim 3 \mathrm{TeV}
$$

but the upper bound in realistic models is usually stronger [39]. Currently the strongest lower bound on $F$ comes from EW precision tests and direct searches for the strong resonances at the LHC, typically resulting in $F>800 \mathrm{GeV}$ [40]. Although one may argue that incalculable UV effects may hinder the impact of EW precision data on compositeness, at this point Higgs data alone can set $F \gtrsim 700 \mathrm{GeV}$ [41].

In the MCHM [15], where $\mathcal{G} / \mathcal{H}=\mathrm{SO}(5) / \mathrm{SO}(4)$, the four pNGBs correspond to the degrees-of-freedom of the Higgs doublet. Larger cosets can provide a stable DM candidate, as shown in refs. [16-18]. In such cases, in order for one of the pseudo-Goldstone bosons to be a viable DM candidate, we must assume that both the strong sector and the SM respect an exact (or approximate) $\mathbb{Z}_{2}$ symmetry, so the lightest $\mathbb{Z}_{2}$-odd pNGB is (quasi-)stable. ${ }^{1}$ Furthermore, since current LHC data points to a SM-like Higgs, we assume the Higgs to be the lightest $\mathbb{Z}_{2}$-even pNGB, transforming as a bi-doublet under $\mathrm{SO}(4)$. On the other hand, the $\mathrm{DM}$ candidate can in general be a mixture of distinct $\mathrm{SO}(4)$-multiplets after electroweak symmetry breaking (EWSB). Here we only consider the cases where the DM state (mostly) belongs to a single $\mathrm{SO}(4)$ multiplet, so the mixing with other multiplets can be ignored. ${ }^{2}$ Therefore, the low energy spectrum consists of the Higgs doublet and the DM multiplet. We assume all possible remaining pNGBs and strong sector resonances to be decoupled in the effective Lagrangian. This approximation is exact in minimal cosets, where the Higgs and the DM multiplet correspond to all the pNGBs, as in the particular examples discussed in section 3. Additionally, note that higher derivative terms in the $\mathcal{G} / \mathcal{H}$ chiral Lagrangian, such as the Wess-Zumino-Witten term [31], can potentially break the $\mathbb{Z}_{2}$ symmetry. Since these terms strongly depend on the $\operatorname{coset} \mathcal{G} / \mathcal{H}$ and the representation of the fermions under $\mathcal{G}$, which we do not specify, they can not be computed in our model independent approach. Hence, we make the simplifying assumption that only those that preserve the $\mathbb{Z}_{2}$ symmetry are present, so the stability of DM is maintained. Furthermore, there are particular choices of cosets $\mathcal{G} / \mathcal{H}$ and fermion representations, where the $\mathbb{Z}_{2}$-violating interactions are zero and the $\xi \rightarrow-\xi$ parity is unbroken, see refs. [16, 17] for a specific realization.

Any composite Higgs model satisfying these assumptions, detailed in the appendix A, can be mapped to a low energy effective theory valid for scales $\ll \Lambda$, as shown schematically by figure 1. Below we discuss the general form of the effective operators relevant for computing the DM observables and their impact on the DM phenomenology.

\footnotetext{
${ }^{1}$ Notice that the $\mathbb{Z}_{2}$ symmetry could already be present within the $\mathcal{G}$ global symmetry group, see e.g. [16].

${ }^{2}$ In models where, after EWSB, there is a significant mixing between the lightest $\mathbb{Z}_{2}$-odd multiplets, the lightest $\mathbb{Z}_{2}$-odd state is usually charged, so there is no viable DM candidate, see e.g. [42] in the noncomposite case.
} 


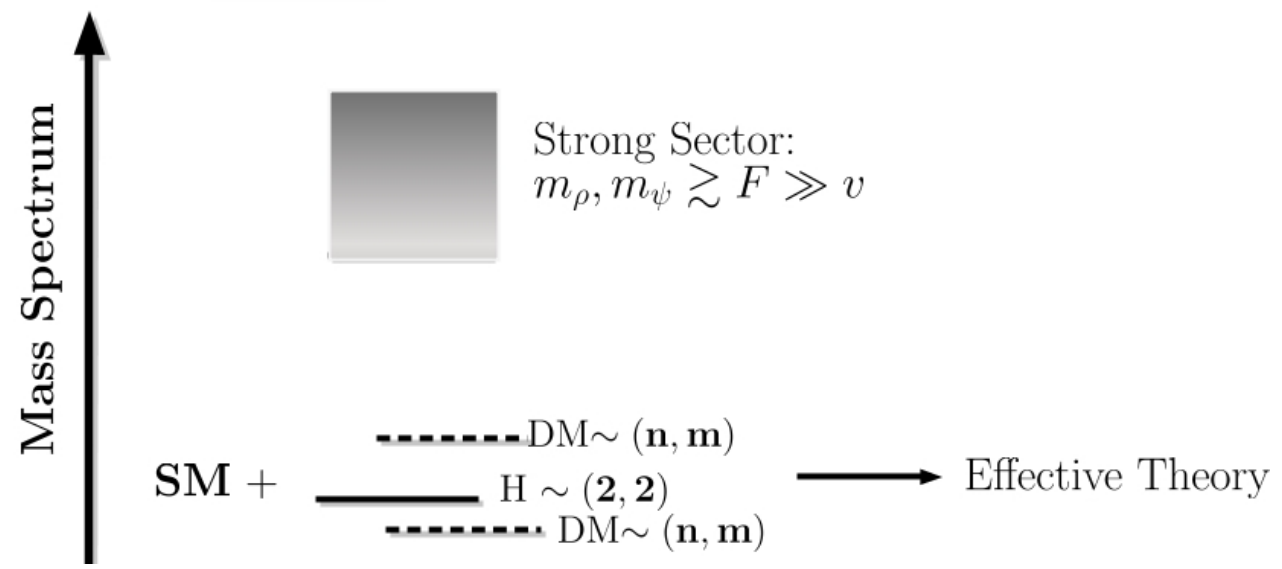

Figure 1. Schematic representation of the spectrum for the class of models considered here. At low energies $(\ll F)$ the effective theory we consider only contains the SM (including the composite Higgs bi-doublet) and the DM multiplet. The two possibilities for the DM mass are shown: $m_{\mathrm{DM}}>m_{h}$ or $m_{\mathrm{DM}}<m_{h}$.

\subsection{Aspects of the effective Dark Matter Lagrangian}

The most general effective Lagrangian consistent with the assumptions discussed in the previous section (see appendix A for more details) contains a large number of unknown parameters. The latter correspond to the coefficients of all the possible dimension six operators involving SM fields as well as those involving the DM multiplet. Fortunately, only a small subset of operators is relevant for computing the DM observables. We also consider the minimal scenario, where DM is a cold thermal relic and the observed DM abundance is generated through the standard freeze-out mechanism. We only consider tree level diagrams in our calculations. In addition, processes where DM annihilates into more than two final states, such as $\xi+\xi \rightarrow X+Y+Z$ are usually phase space suppressed with respect to $\xi+\xi \rightarrow X+Y$. Hence we neglect operators with more than four fields. Notice though that in some cases, annihilation into three body final states are well known to be important [43-47]. In particular, here we will take into account the contribution of annihilations into off-shell gauge bosons $\xi+\xi \rightarrow V+V^{*}$. Let us also emphasize that dimension five and six operators can become relevant if they contain one or more Higgs fields, since after EWSB these operators generate 4-field operators (with $H \rightarrow\langle H\rangle=v$ ). Furthermore, the $\mathbb{Z}_{2}$ symmetry, under which $\xi$ is odd and $H$ is even, requires all operators to contain an even number of $\xi$ 's.

Given all the assumptions above, the operators containing more than two powers of $\xi$ do not affect the relic abundance or the detection rates. We just need to consider operators with zero or two DM fields. Hence, for convenience, we split the effective DM Lagrangian in a $\xi$-independent sector $\left(\mathcal{L}^{(0)}\right)$ and a part containing two powers of $\xi\left(\mathcal{L}^{(2)}\right)$ :

$$
\mathcal{L}_{\text {eff }}=\mathcal{L}^{(0)}(H, \ldots)+\mathcal{L}^{(2)}(H, \xi, \ldots),
$$

with $\mathcal{L}^{(0)}$ given by

$$
\mathcal{L}^{(0)}(H, \ldots)=\mathcal{L}_{\mathrm{SM}}+\mathcal{L}_{6},
$$


where $\mathcal{L}_{\mathrm{SM}}$ is the $\mathrm{SM}$ Lagrangian and $\mathcal{L}_{6}$ contains the relevant dimension-6 operators induced by the strong dynamics. Since $\mathcal{L}_{6}$ is independent of $\xi$, its operators are common for all the cases to be discussed later. The SM dimension- 6 operators have been explored at length in the literature [48-53] and are briefly discussed in appendix A. Below we simply list the higher dimensional operators considered in our analyses. We use

$$
\mathcal{L}_{6}=\frac{a_{2 H}}{F^{2}}\left(\partial_{\mu}|H|^{2}\right)^{2}-\frac{\lambda_{1} \lambda_{H 6}}{F^{2}}|H|^{6}-\frac{c_{4}}{F^{2}}|H|^{2}\left[\left(y_{t} \bar{Q}_{L} H^{c} t_{R}+y_{b} \bar{Q}_{L} H b_{R}\right)+\text { h.c. }\right],
$$

where $a_{2 H}, c_{4}$ and $\lambda_{H 6}$ are generated after we integrate out the strong sector, while $\lambda_{1}$ is the dim- 4 Higgs self-coupling. As we assume a $C P$-even Higgs, $c_{4}$ is real. The coefficients $a_{2 H}, c_{4}$ and $\lambda_{H 6}$ are considered as $\mathcal{O}(1)$ parameters. Notice that the operators in eq. (2.4) are weakly constrained by the LHC data $[49,54]$.

In order to reduce the number of parameters that would potentially affect the DM observables and simplify our analysis, we consider one single coefficient $c_{4}$ multiplying the dimension- 6 operators involving a direct coupling between the Higgs particle and the top and bottom quarks. As far as relic abundance and indirect dark matter detection searches are concerned, our choice could be justified by the fact that this extra coupling to top and bottom quarks are relevant in different regions of the parameter space. The impact of similar operators involving light quarks would be suppressed by the Yukawas $\left(y_{\psi}\right)$. Let us emphasize though that this is however not true for direct dark matter searches in which case the relevant Higgs-nucleon coupling is dominated by heavy quarks $(b, t$ and $c$ ) contributions. The latter are due to heavy quark currents coupling to gluons through triangle diagrams involving heavy quark loops, see e.g. [55] and references therein. ${ }^{3}$

As it is well known, after EWSB the operator proportional to $a_{2 H}$ generates a noncanonical kinetic term for the Higgs field. The rescaling to a physical Higgs introduces the factor

$$
R=1 / \sqrt{1+2 a_{2 H} \frac{v^{2}}{F^{2}}}
$$

in all Higgs interactions. Even though we include $R$ in our numerical calculations, for simplicity we will typically neglect it in the analytic expressions. Indeed $R$ is $\simeq 1$ for $F \gtrsim 1 \mathrm{TeV}$.

Notice that we do not consider the dimension- 6 operators that modify the gauge couplings to fermions, such as $\left(H^{\dagger} \sigma^{j} \overleftrightarrow{D}^{\mu} H\right)\left(\bar{Q}_{L} \sigma^{j} \gamma_{\mu} Q_{L}\right)$, and those which contribute to the electroweak parameters at tree level, like $\left(H^{\dagger} \sigma^{j} H\right)\left(W_{\mu \nu}^{j} B^{\mu \nu}\right)$ [48-52]. As it is well known, these operators are strongly constrained by the electroweak fit [40].

Since we assume that $\xi$ is a $\mathrm{SO}(4) \sim \mathrm{SU}(2)_{L} \times \mathrm{SU}(2)_{R}$ multiplet, the possible representations for $\xi$ are

$$
\left(2 j_{L}+1,2 j_{R}+1\right)=(1,1),(2,2),(3,1),(1,3),(3,3),(2,4), \ldots
$$

\footnotetext{
${ }^{3}$ Let us mention though that the contribution to the amplitude of DM-nucleon scattering related to the coupling $c_{4}$ is always expected to be a subdominant correction compared to other operators as it appears in the following combination: $\lambda_{\xi H}\left(1+c_{4} v^{2} / F^{2}\right)$ (where $\lambda_{\xi H}$ is the Higgs-DM coupling). We expect thus the impact of choosing a common dimension- 6 coefficient $c_{4}$ to all quarks to be marginal.
} 
In the following we limit our results to the cases

- Singlet DM: $\xi \sim(1,1)$

- Doublet DM: $\xi \sim(2,2)$

- Real Multiplet DM $\left(\xi^{C}=\xi\right): \xi \sim(n, 1)$, where $n=3,5, \ldots$.

Our restriction to real representations (except in the doublet case) is justified by the fact that the phenomenology associated to complex multiplets would be very similar to the real multiplet case; the complex multiplet being equivalent to two degenerate real multiplets. A real DM multiplet also implies that $\xi$ is not charged under any $\mathrm{U}(1)$ symmetry, since $\xi^{C}=C \xi^{*}=\xi$. Consequently, $\xi$ is not charged under $\mathrm{U}(1)_{Y}$ and since the hypercharge operator is a linear combination of $T_{R}^{3} \subset \mathrm{SU}(2)_{R}$ and $\mathrm{U}(1)_{X},{ }^{4}$ it means that $\xi$ is a singlet under $\mathrm{SU}(2)_{R}$. Hence $\xi \sim(n, 1)$.

Finally we briefly comment on the validity region of the effective Lagrangian used in our analysis. The energy involved in the relevant DM processes is typically $\sqrt{s} \lesssim 2 m_{\text {DM }}$, where the upper limit corresponds to non-relativistic annihilation at zero velocity today. In the early universe, energies involved are usually slightly larger given that $v \sim 0.2$ while for direct searches the energies involved are much smaller. Therefore, we expect the effective Lagrangian approach used here to be valid as long as the new physics states (which could affect the DM observables) are well above $2 m_{\mathrm{DM}}$. In composite Higgs models one naively expects the strong resonances to have masses of order $\Lambda \simeq 4 \pi F$. However, the composite partners of the top quark must be lighter in order to avoid re-introducing a large finetuning in the Higgs potential [39]. Here we assume that the spinorial resonances have masses $m_{\Psi} \gtrsim F$, hence our effective Lagrangian is valid for $m_{\mathrm{DM}} \ll m_{\Psi}$ or $m_{\mathrm{DM}} \ll F$, which we use as a strict upper limit in our study. It is also important to point out that, for $m_{\mathrm{DM}}<F$, all the operators used in our analysis are far away from any unitarity bounds $[57,58]$.

\subsection{The effect of higher dimensional operators}

The specific form of the dimension- 4 and 6 relevant operators ${ }^{5}$ consistent with $\mathrm{SO}(4) \subset \mathcal{H}$ are derived in detail in the appendix A and will be discussed in section 3. It is instructive, however, to first comment on the general properties of these operators and how they can affect the DM observables. Since we are only interested in 4-field operators after EWSB, the relevant dimension- 6 operators generated by integrating out the strong sector heavy resonances are of the following types

$$
\frac{y_{f}}{v} \frac{v^{2}}{F^{2}} \xi^{2} \bar{\psi} \psi, \lambda \frac{v^{3}}{F^{2}} \xi^{2} h, \lambda \frac{v^{2}}{F^{2}} \xi^{2} h^{2}, g^{2} \frac{v^{2}}{F^{2}} \xi^{2} V_{\mu} V^{\mu}, g \frac{v p_{\mu}}{F^{2}} V_{\mu} h, \frac{v p^{2}}{F^{2}} \xi^{2} h \text { and } \frac{p^{2}}{F^{2}} \xi^{2} h^{2},
$$

where $h$ is the Higgs boson field, $V_{\mu}$ represents a $\mathrm{SU}(2)_{L} \times \mathrm{U}(1)_{Y}$ gauge field, $\psi$ is a $\mathrm{SM}$ fermion, $g$ is the corresponding gauge coupling, $\lambda$ represents the corresponding dimension- 4

\footnotetext{
${ }^{4} \mathrm{As}$ it is well known in composite Higgs models, it is often necessary to enlarge the $\mathcal{G}$ group with an additional $\mathrm{U}(1)_{X}$ symmetry in order to obtain the correct hypercharges for the SM fermions $[48,56]$.

${ }^{5}$ We always mean by dimension- 6 operators, the operators suppressed by $1 / F^{2}$.
} 
coupling and $p_{\mu}$ refers to the typical momentum scale for the process. As all the above operators are presumed to have coefficients $\lesssim 1$, one would expect such operators to be irrelevant with respect to the corresponding dimension-4 operators for $F \gg v$. However it is often the case that, in order to generate the correct relic abundance, the dimension4 operators coefficients need to be suppressed. In such cases, the dimension- 6 operators may still play a significant role. Furthermore, the derivative operators can be enhanced if $p^{2} \sim m_{\mathrm{DM}}^{2} \gg v^{2}$.

In order to illustrate the above arguments more concretely, we take the singlet DM case as an example. In the non-composite limit $(F \rightarrow \infty)$, there is only one relevant dimension-4 operator, corresponding to the Higgs-DM coupling $\left(\lambda_{\xi h}\right)$, also known as the Higgs portal coupling. As a result, the parameter space in the non-composite singlet case consists only of $\lambda_{\xi h}$ and $m_{\mathrm{DM}}$. As it is well known, requiring the DM relic energy density $\Omega_{\mathrm{DM}} h^{2} \simeq 0.12[26,59]$ fixes the Higgs-DM coupling for a given DM mass and the solutions with the correct relic abundance correspond to a line in the $\lambda_{\xi h}-m_{\mathrm{DM}}$ plane. This is shown by the black solid line in figure 2, where we plot the value of the Higgs-DM coupling in the non-composite case $\left(\lambda_{\xi h}^{\mathrm{NC}}\right)$ required to generate the correct relic abundance. As we can see, $\lambda_{\xi h}^{\mathrm{NC}}$ does indeed take values $\ll 1$ in most of the parameter space, which may render the operators in eq. (2.7) relevant in these regions even though they are suppressed by $1 / F^{2}$. In order to estimate for which values of $m_{\mathrm{DM}}$ the inclusion of the dimension- 6 operators may affect the DM observables, we compare their potential effective strength with respect to $\lambda_{\xi h}^{\mathrm{NC}}$.

Assuming that the coefficients multiplying the operators in eq. (2.7) are $\mathcal{O}(1)$, the blue shaded areas in figure 2, for $F=0.8 \mathrm{TeV}$ (left) and $F=2.5 \mathrm{TeV}$ (right), correspond to the windows of the parameter space in which these operators can potentially drive the relic abundance. In the regions below the continuous horizontal blue line $\lambda_{\xi h}^{\mathrm{NC}} \lesssim v^{2} / F^{2}$, i.e. the non-derivative operators can be dominant, while the regions below the blue dotted curves $\lambda_{\xi h}^{\mathrm{NC}} \lesssim 4 m_{\mathrm{DM}}^{2} / F^{2}$, i.e. the derivative operators can become dominant. As we can see, for $F=0.8 \mathrm{TeV}$, the dimension- 6 operators can potentially affect the DM cross-sections for $m_{\mathrm{DM}} \gtrsim 35 \mathrm{GeV}$. On the other hand, for higher values of $F$ this contribution becomes limited to the Higgs resonance, where $\lambda_{\xi h}^{\mathrm{NC}} \ll 1$, or the heavy DM region, where the derivative operators can be enhanced. Furthermore, for $F=2.5 \mathrm{TeV}$, we see that the non-derivative operators play no role, except around the resonance. We stress that the results shown in figure 2 are purely schematic and are intended only to illustrate the potential impact of including the higher dimensional operators in our analyses. However, as it will be shown in the next section, the overall features described above do appear in our numerical computations.

It is also interesting to point out that the dimension- 6 operators can have very distinct contributions to the annihilation and nucleon scattering rates. On the one hand, while the momentum transferred in DM-nucleon scattering is $\mathcal{O}(\mathrm{keV}-\mathrm{MeV})$, the momentum scale involved in DM annihilations in the early universe is of order $m_{\mathrm{DM}}$. Hence, the derivative operators which scale as $p^{2} / F^{2}$ only contribute significantly to DM annihilations and not to the direct detection rate. On the other hand, dimension-6 operators involving Yukawa 

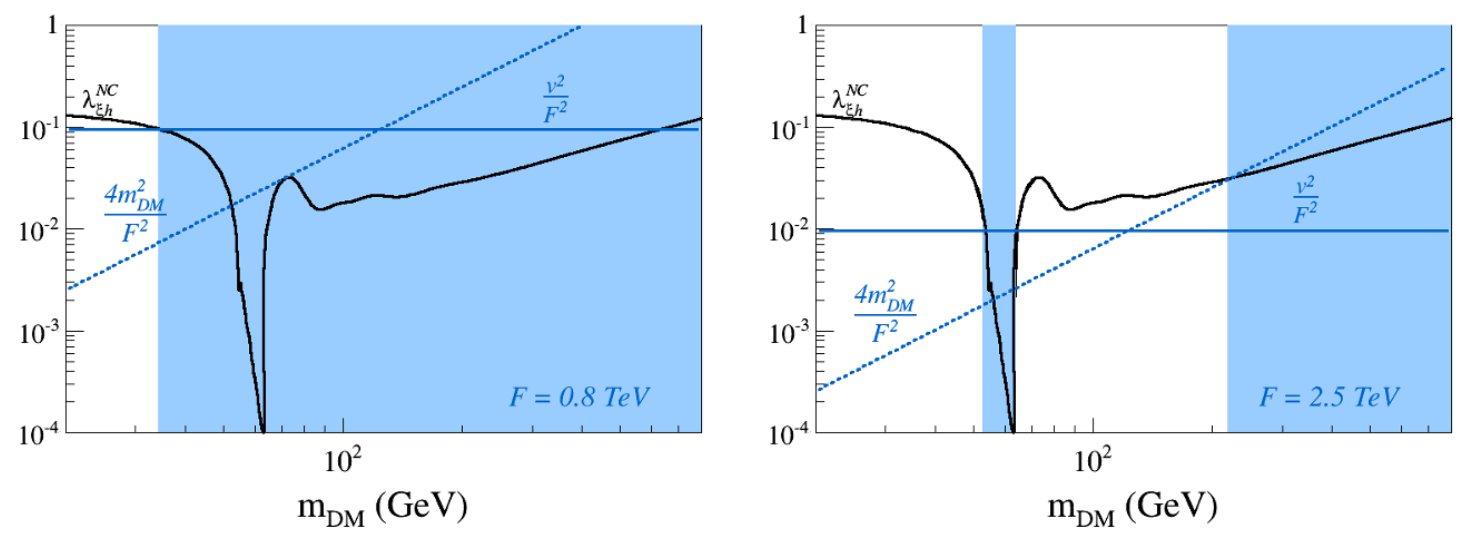

Figure 2. Singlet DM model: the black continuous line denotes the values of the DM-Higgs coupling in the non-composite case $\left(\lambda_{\xi h}^{\mathrm{NC}}\right)$ as a function of $m_{\mathrm{DM}}$ giving rise to the right relic abundance. The blue shaded areas correspond to the windows of the parameter space in which the dimension6 operators of eq. (2.7) can potentially affect the DM observables for $F=0.8 \mathrm{TeV}$ (left) and $F=2.5 \mathrm{TeV}$ (right). In the regions below the continuous horizontal blue line $\lambda_{\xi h}^{\mathrm{NC}} \lesssim v^{2} / F^{2}$ while in the regions below the blue dotted curves $\lambda_{\xi h}^{\mathrm{NC}} \lesssim 4 m_{\mathrm{DM}}^{2} / F^{2}$.

type of interactions such as

$$
\frac{C_{q}}{F^{2}}|\xi|^{2}\left(y_{q} \bar{Q}_{L} H^{c} q_{R}\right)
$$

where $y_{q}$ is the Yukawa coupling of the $q$-quark and $C_{q}$ a coefficient of $\mathcal{O}(1)$, give important contributions to the dark matter-nucleon scattering cross section, $\sigma_{\mathrm{DMp}}$, for $q=c, b$ and $t$ (through triangle diagrams processes that are, by default, taken into account using dark matter tools such as micrOMEGAS, see [60]).

Let us mention that, in the following, we have considered interactions of the type of (2.8) with couplings to the $3 \mathrm{rd}$ generation of quarks only $\left(C_{u, d, c, s}=0\right)$. This assumption does not affect the relic density and the indirect detection results, since the contributions from light quarks is always suppressed by their Yukawas. The couplings to light quarks can however impact $\sigma_{\mathrm{DMp}}$, especially in the regions where $\lambda_{\xi h}$ is small. Given the values of the parameters driving the nucleon matrix element considered in our analysis, ${ }^{6}$ we expect that including a coupling to all quarks would at most enhance $\sigma_{\mathrm{DMp}}$ by a factor of $\sim 4$ and only if $\left|\lambda_{\xi h}\right| \ll m_{h}^{2} / F^{2}\left|C_{q}\right|$ (see also [17] for a similar discussion). In order to reduce the number of free parameters in our analysis, we have however neglected this possibility. Finally, we make one additional simplifying assumption: $C_{t}=C_{b}$. Once again, this assumption does not affect the relic density or direct detection rate, since annihilations to tops or bottoms are relevant at distinct regions of parameter space. While $\sigma_{\mathrm{DMp}}$ could change for nonuniversal couplings, we do not expect that our results are significantly affected by this assumption, since we expect $C_{t} \sim C_{b} \lesssim \mathcal{O}(1)$.

\footnotetext{
${ }^{6}$ We took the default values of micrOMEGAS3.3 corresponding to $f_{d}^{p}=0.0191, f_{u}^{p}=0.0153$ and $f_{s}^{p}=0.0447$ in the notations of ref. [60].
} 


\section{Results}

Using the effective Lagrangians derived in appendix A, we now compute the DM observables and discuss how this constrains the effective operators for a given DM representation. The DM relic abundance and other observables have been computed using FeynRules [61] and micrOMEGAS [62]. The calculation of the relic abundance includes all possible annihilation channels into two body as well as annihilation into off-shell $V=W$ or $Z$ boson $(\xi+\xi \rightarrow$ $\left.V^{*}+V\right)$, which can be relevant for $m_{\mathrm{DM}} \lesssim m_{V}$. Even though radiative corrections are already known to affect DM observables in similar non-composite DM scenarios, especially the dark matter-nucleon scattering cross section [63-65], it is beyond the scope of this paper to evaluate all loop contributions within the composite framework (the exception being the well known contributions from triangle diagrams of heavy quark loops included by default in micrOMEGAS for the computation of the DM-nucleon scattering cross section). One should keep in mind though that such corrections are expected to give rise to the lower bound $\sigma_{D M p} \gtrsim 10^{-47} \mathrm{~cm}^{2}$ for non-composite models in e.g. the doublet case [64].

In order to be as model independent as possible, we parametrize the effective Lagrangian according to the results in appendix A and scan over the $\mathcal{O}(1)$ coefficients of the dimension- 6 operators in the range $[-1,1]$. The unknown parameters for effective dimension- 4 operators (denoted with $\lambda$ couplings) in the DM Lagrangian are also allowed to vary freely within a range restricted to $[-4 \pi, 4 \pi]$. We also require all the solutions to satisfy: $0.0941<\Omega_{\mathrm{DM}} h^{2}<0.127 .{ }^{7}$

In addition to the above conditions, directly taken into account in the scans, we will superimpose several constraints from LHC, direct and indirect DM searches. Furthermore, the low DM mass region is limited by the Higgs invisible width, which has been recently constrained by ATLAS [24] and CMS [25]. Here we use the 95\% C.L. upper limit from CMS: $\operatorname{BR}(h \rightarrow$ invisible $)<0.58$. For the direct detection constraints, we consider the latest LUX results [19] on the spin-independent DM-nucleon elastic cross-section as well as the projected sensitivity of Xenon1T, both at $95 \% \mathrm{CL}$. The DM annihilation into SM particles in our galaxy could copiously produce stable particles, such as photons, neutrinos, positrons or antiprotons. In that framework, indirect detection searches constraints from Fermi-LAT $[20,21]$ and PAMELA $[67,68]$ can be relevant here. Indeed, a previous analysis of composite DM models [17] showed that the bound on the DM annihilation cross-section derived from PAMELA measurements of the antiproton spectrum could provide constraints complementary to LHC and direct detection searches. The bounds associated to charged cosmic rays are, however, well known to suffer from astrophysical uncertainties [69, 70] affecting the propagation model. Here we use the $3 \sigma$ upper bound constraints derived in ref. [70] (figure 6c) for the DM annihilation cross-section into $\bar{b} b .{ }^{8}$ Let us mention that such a bound has been derived assuming an uncertainty of $50 \%$ in the solar modulation of the antiproton flux and we take the limits associated to the so called CON propagation model

\footnotetext{
${ }^{7}$ We used the $2 \sigma$ WMAP 5-year range [66]. For a reference, the $3 \sigma$ WMAP 9-year range is $0.1003<$ $\Omega_{\mathrm{DM}} h^{2}<0.1273$ [59] and the $3 \sigma$ PLANCK range is $0.1103<\Omega_{\mathrm{DM}} h^{2}<0.1289$ [26].

${ }^{8}$ The PAMELA constraints are thus imposed on $\left.\sigma v(\xi \xi \rightarrow \bar{b} b)\right|_{v=0}$ rescaling the constraints of ref. [70] by the associated branching ratio.
} 
(green curve in figure 6c of ref. [70]), which usually corresponds to conservative bounds. Let us emphasize that even though similar bounds were derived for other annihilation channels such as e.g. $\xi \xi \rightarrow W^{+} W^{-}$, we checked that they provide weaker constraints than the direct detection searches. We have thus neglected them. Let us also mention that the current preliminary constraints on gamma-ray flux from the Fermi-LAT [21] measurements provide even stronger bounds on $\left.\sigma v(\xi \xi \rightarrow \bar{b} b)\right|_{v=0}$. We have checked that the impact of these preliminary results are negligible, so we only consider the constraints from PAMELA.

The LHC constraints from monojet and monophoton searches are still insufficient to compete with the direct detection or invisible Higgs decay searches. Nonetheless, for both the doublet and triplet representations, the DM multiplet contains charged states, which can decay to DM and be detectable at the LHC. The main final states are then missing $E_{T}$ plus $W$ 's, Z's and/or Higgses. Using SModelS [71] we were able to recast the LHC constraints for SUSY searches and apply them to the scenarios considered. We have explicitly computed the relevant production cross-sections and verified that the LHC constraints do not lead to any new excluded region. Therefore we will not consider them in the following results.

\subsection{Singlet DM}

\subsubsection{The generic composite singlet DM}

The singlet DM case corresponds to the following effective Lagrangian (for details see appendix A.1)

$$
\begin{aligned}
\mathcal{L}^{(2)}= & \frac{1}{2} \partial_{\mu} \xi \partial^{\mu} \xi-\frac{1}{2} \mu_{\xi}^{2} \xi^{2}-\frac{\lambda_{3}}{2}\left(1+\frac{\lambda_{3}^{\prime}}{F^{2}}|H|^{2}\right) \xi^{2}|H|^{2}+\frac{a_{d 1}}{F^{2}} \partial_{\mu} \xi^{2} \partial^{\mu}|H|^{2} \\
& -\frac{1}{2}\left[\frac{d_{4}}{F^{2}} \xi^{2}\left(y_{t} \bar{Q}_{L} H^{c} t_{R}+y_{b} \bar{Q}_{L} H b_{R}\right)+\text { h.c. }\right],
\end{aligned}
$$

resulting in the following DM mass after EWSB:

$$
m_{\mathrm{DM}}^{2}=\mu_{\xi}^{2}+\lambda_{3}\left(1+\frac{\lambda_{3}^{\prime}}{2} \frac{v^{2}}{F^{2}}\right) \frac{v^{2}}{2} .
$$

The coefficients $a_{d 1}, d_{4}, \lambda_{3}^{\prime}$ are taken to be real $\mathcal{O}(1)$ parameters, while the $\lambda_{3}$ coupling is allowed to vary in the window $[-4 \pi, 4 \pi]$ and $\mu_{\xi}$ is the DM bare mass. Except for $a_{d 1}$, which is fixed by $\mathcal{G} / \mathcal{H}$, all the other coefficients depend on the fermion embedding in $\mathcal{G}$. Therefore in our model independent approach we take these to be free parameters. As discussed in section 2.2, we neglect dimension-6 operators involving light quarks and we assume a universal coefficient $\left(d_{4}\right)$ for the top and bottom couplings.

For $m_{\mathrm{DM}}<m_{t}$, the DM observables are mostly determined by the effective $\xi\left(p_{1}\right)-$ $\xi\left(p_{2}\right)-h\left(p_{h}\right)$ coupling, which in the composite case is given by:

$$
\lambda_{\xi h}=\left(\frac{\bar{\lambda}}{2}-a_{d 1} \frac{p_{h}^{2}}{F^{2}}\right),
$$

where

$$
\bar{\lambda}=\lambda_{3}\left(1+\lambda_{3}^{\prime} \frac{v^{2}}{F^{2}}\right)
$$

and $p_{h}^{2}$ is the (off-shell) Higgs momentum. 
It is important to point out that the effective DM-Higgs coupling relevant for the direct detection (DD) rate and the relic density $(\mathrm{RD})$ (or $\left\langle\sigma_{\mathrm{ann}} . v\right\rangle$ ) can take very different values, due to the momentum dependence in eq. (3.3). While for annihilations through the Higgs $s$-channel we have $p_{h}^{2} \simeq 4 m_{\mathrm{DM}}^{2}$, for direct detection we have $t$-channel scattering, hence $p_{h}^{2} \simeq 0$. Therefore it is convenient to define the effective couplings in each of these regimes:

$$
\begin{aligned}
\left.\lambda_{\xi h}\right|_{p_{h} \sim 2 m_{\mathrm{DM}}} & \equiv \lambda_{\xi h}^{R D}=\frac{\bar{\lambda}}{2}-4 a_{d 1} \frac{m_{\mathrm{DM}}^{2}}{F^{2}}, \\
\left.\lambda_{\xi h}\right|_{p_{h} \sim 0} & \equiv \lambda_{\xi h}^{D D}=\frac{\bar{\lambda}}{2},
\end{aligned}
$$

where $\lambda_{\xi h}^{R D}\left(\lambda_{\xi h}^{D D}\right)$ is the relevant coupling for the calculation of the relic abundance (DD cross-section).$^{9}$

In the low mass region, where annihilations to tops are closed, both the DM-nucleon spin independent scattering cross-section, $\sigma_{\mathrm{DMp}}$, and the thermally averaged annihilation cross-section times the relative velocity at the freeze-out time, $\left\langle\sigma_{\mathrm{ann}} \cdot v\right\rangle$, are proportional to the Higgs-DM effective coupling:

$$
\begin{aligned}
\left\langle\sigma_{\mathrm{ann}} \cdot v\right\rangle & \propto\left(\lambda_{\xi h}^{R D}\right)^{2} \simeq\left(\frac{\bar{\lambda}}{2}-4 a_{d 1} \frac{m_{\mathrm{DM}}^{2}}{F^{2}}\right)^{2}, \\
\sigma_{\mathrm{DMp}} & \propto\left(\lambda_{\xi h}^{D D}\right)^{2} \simeq\left(\frac{\bar{\lambda}}{2}\right)^{2} .
\end{aligned}
$$

In analogy to the non-composite case, once we impose $\Omega_{\mathrm{DM}} h^{2} \simeq 0.12, \lambda_{\xi h}^{R D}$ is fixed for each value of $m_{\mathrm{DM}}$. Denoting by $\lambda_{\xi h}^{\mathrm{NC}}$ the value of the coupling required to generate the correct relic abundance in the non-composite case (shown by the black curve in figure 2), we have:

$$
\begin{aligned}
\left(\lambda_{\xi h}^{R D}\right)^{2} \simeq\left(\lambda_{\xi h}^{\mathrm{NC}}\right)^{2} & \Rightarrow\left(\frac{\bar{\lambda}}{2}\right)^{2} \simeq\left(\lambda_{\xi h}^{\mathrm{NC}} \pm 4 a_{d 1} \frac{m_{\mathrm{DM}}^{2}}{F^{2}}\right)^{2} \\
& \Rightarrow \sigma_{\mathrm{DMp}} \propto\left(\lambda_{\xi h}^{\mathrm{NC}} \pm 4 a_{d 1} \frac{m_{\mathrm{DM}}^{2}}{F^{2}}\right)^{2} \text { or } \sigma_{\mathrm{DMp}} / \sigma_{\mathrm{DMp}}^{\mathrm{NC}}=\left(1 \pm 4 \frac{a_{d 1}}{\lambda_{\xi h}^{\mathrm{NC}}} \frac{m_{\mathrm{DM}}^{2}}{F^{2}}\right)^{2}
\end{aligned}
$$

where $\sigma_{\mathrm{DMp}}^{\mathrm{NC}}$ is the DM-nucleus cross-section in the non-composite case. From the above result we see that $\sigma_{\mathrm{DMp}}$ can be either enhanced or suppressed with respect to its noncomposite value, depending on the values of $a_{d 1}$ and $m_{\mathrm{DM}}$. Let us stress that the above results are only approximate, since we have neglected the contributions from $d_{4}$, both for relic abundance and direct detection considerations. The impact of $d_{4}$ is, however, included in our numerical analysis.

\subsubsection{Scan results}

We now present the full numerical results for the DM observables for a random scan over the coefficients of the effective Lagrangian. Since in some cases the correct relic abundance requires extremely small values of $\lambda_{3}, \lambda_{3}^{\prime}$ and $\lambda_{H 6}$, we scan logarithmically over their allowed

\footnotetext{
${ }^{9}$ Let us mention that, since we have not considered DM contact interactions with light quarks, the $d_{4}$ coupling has only a minor impact on the general DD picture. If couplings to light quarks were included, the results could be modified in some small regions of parameter space, where $\left|\lambda_{\xi H}\right| \ll\left|d_{4}\right| m_{h}^{2} / F^{2}$, see section 2.2 for a brief discussion.
} 

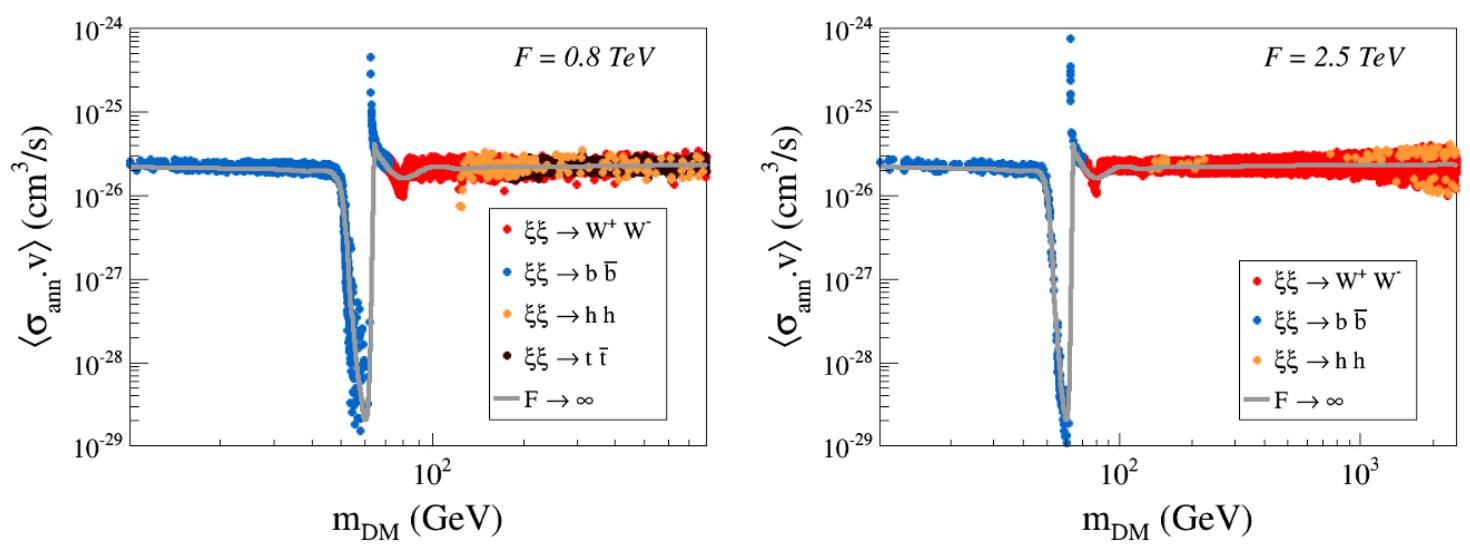

Figure 3. Generic composite singlet $D M$ : values of the DM annihilation cross-section at zero velocity for $F=0.8 \mathrm{TeV}$ (left) and $F=2.5 \mathrm{TeV}$ (right) satisfying to $0.941<\Omega_{\mathrm{DM}} h^{2}<0.127$. The channels giving rise to the largest branching ratio to the annihilation cross-section are shown with different colors. We also show as a solid gray line the value of $\left\langle\sigma_{\mathrm{ann}} . v\right\rangle$ for the non-composite case $(F \rightarrow \infty)$.

range. All the other parameters are scanned linearly within the ranges:

$$
\begin{aligned}
10 \mathrm{GeV} & <m_{\mathrm{DM}}<F, \\
-4 \pi & <\lambda_{3}<4 \pi, \\
-1 & <\lambda_{3}^{\prime}<1, \\
10^{-6} & <\lambda_{H 6}<1, \\
-1 & <c_{4}, a_{2 H}, d_{4}, a_{d 1}<1 .
\end{aligned}
$$

First, in figure 3, we show the results for the DM annihilation cross-section $\left(\left\langle\sigma_{\text {ann }} \cdot v\right\rangle\right)$ today at zero velocity as a function of $m_{\mathrm{DM}}$. For comparison purposes we also show the respective values for $\left\langle\sigma_{\mathrm{ann}} \cdot v\right\rangle$ in the non-composite case (solid gray line). As we can see, for both $F=0.8 \mathrm{TeV}$ (left plot) and $2.5 \mathrm{TeV}$ (right plot), the annihilation rate follows closely the non-composite case, as expected from the relation $\Omega_{\mathrm{DM}} h^{2} \propto 1 /\left\langle\sigma_{\mathrm{ann}} \cdot v\right\rangle$. We notice, however, that while in the $F=2.5 \mathrm{TeV}$ case the dominant annihilation channels are the same as in the non-composite case $\left(\xi \xi \rightarrow \bar{b} b\right.$ or $\left.\xi \xi \rightarrow W^{-} W^{+}\right)$, for $F=0.8 \mathrm{TeV}$, both annihilations to $\bar{t} t$ and $h h$ can be dominant at large DM masses. This is mostly due to the fact that, for small values of $F$, the non-derivative operators $d_{4} \xi^{2} \bar{Q}_{L} H^{c} t_{R}$ can be comparable to $\lambda_{\xi h}$, as schematically shown in the left hand (LH) side plot of figure 2. On the other hand, for $F=2.5 \mathrm{TeV}$, these operators are always suppressed.

The DM-nucleon scattering cross-section $\left(\sigma_{\mathrm{DMp}}\right)$ is shown as a function of $m_{\mathrm{DM}}$ in figure 4 for $F=0.8 \mathrm{TeV}$ (left) and $2.5 \mathrm{TeV}$ (right). All points give the correct DM relic abundance. Once again the non-composite case is shown by the solid gray line. Going from low to high $m_{\mathrm{DM}}$ it is possible to recognize in figure 4 some known features $[16,17]$ : the Higgs resonance region $\left(m_{\mathrm{DM}}=m_{h} / 2\right)$, the $\xi \xi \rightarrow W W$ threshold $\left(m_{\mathrm{DM}} \simeq m_{W}\right)$ and the high mass region $\left(m_{\mathrm{DM}} \gtrsim m_{t}\right)$. We also show by distinct colors the absolute value of the coefficient $a_{d 1}$ for each point. As we can see, the low mass region $\left(m_{\mathrm{DM}} \lesssim 50 \mathrm{GeV}\right)$ is unaffected by the dimension- 6 operators induced by the strong sector. This is already 

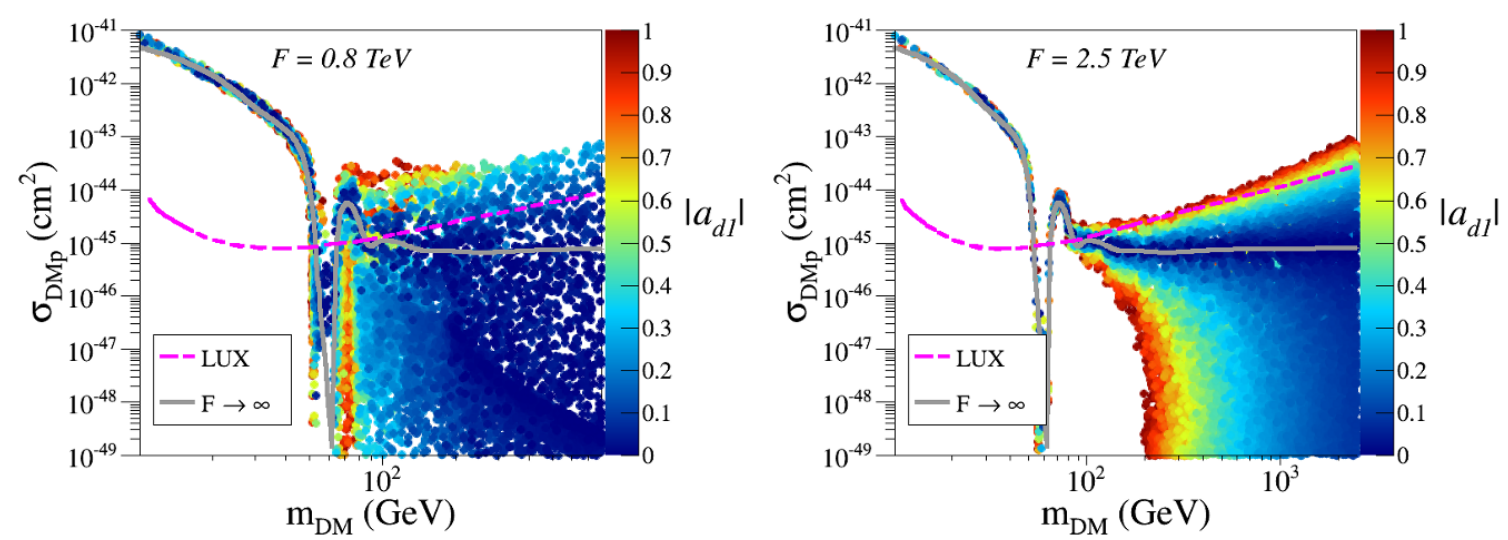

Figure 4. Generic composite singlet DM: values of the DM-nucleon scattering cross-section as a function of $m_{\mathrm{DM}}$. All points satisfy $0.0941<\Omega_{\mathrm{DM}} h^{2}<0.127$. The absolute values of the dimension6 coefficient $a_{d 1}$ are shown with the gradient of colors. The LUX 95\% CL bound is also shown.

expected from the discussion in section 2.2, where we have shown that the low mass region is mostly insensitive to these operators.

Once $m_{\mathrm{DM}} \gtrsim 50 \mathrm{GeV}, \sigma_{\mathrm{DMp}}$ can take values drastically different from the non-composite case. As expected from eq. (3.7), this is due to the contribution of the derivative operator proportional to $a_{d 1}$ (at least for $m_{\mathrm{DM}} \lesssim m_{t}$ ). Indeed, given that we can get the right abundance with $4 a_{d 1} m_{\mathrm{DM}}^{2} / F^{2} \simeq \lambda_{\xi h}^{\mathrm{NC}}$, i.e. for small values of $\bar{\lambda}, \sigma_{\mathrm{DMp}}$ can become very suppressed, as seen in figure 4 . In addition, since $a_{d 1}$ can take both negative and positive values, we can also have the correct DM abundance with $\bar{\lambda}$ larger than in the non-composite case, resulting in an enhancement of $\sigma_{\mathrm{DMp}}$, as shown by the points above the gray line in figure 4 .

For $m_{\mathrm{DM}}>m_{t}$, annihilations to top pairs become kinematically allowed and $\left\langle\sigma_{\mathrm{ann}} \cdot v\right\rangle$ receives a contribution from the operator proportional to $d_{4}$. In this case, we can once again generate the correct relic abundance, even when $\lambda_{\xi h} \ll \lambda_{\xi h}^{\mathrm{NC}}$. This corresponds to all the points at large masses with small values of $\sigma_{\mathrm{DMp}}$ and $a_{d 1}$ in the LH side plot of figure 4. Notice though that this feature is not as strongly present in the right hand $(\mathrm{RH})$ side plot of figure 4 for $F=2.5 \mathrm{TeV}$. This is simply due to the fact that for such large $F$ values and $m_{\mathrm{DM}}>m_{t}$, non-derivative operators, such as the one proportional to $d_{4}$, are always subdominant, as already anticipated by the RH side plot of figure 2. Finally, we also emphasize that for $F=2.5 \mathrm{TeV}, \sigma_{\mathrm{DMp}}$ only deviates significantly from its non-composite value for higher DM masses $\left(m_{\mathrm{DM}} \gtrsim 150 \mathrm{GeV}\right)$, as expected from eq. (3.7) and in agreement with the discussion in section 2.2. From figure 4 we already see that for $m_{\mathrm{DM}}>200 \mathrm{GeV}$ to be consistent with LUX we need $\left|a_{d 1}\right| \lesssim 0.2$ for $F=0.8 \mathrm{TeV}$, higher values are however possible if we increase the scale of compositeness.

\subsubsection{Experimental constraints}

We now discuss the current and future constraints on the generic composite singlet DM scenarios. As seen in figure 4, the direct detection cross-section can be significantly suppressed or enhanced in the composite case. Nonetheless, the low mass region still behaves 

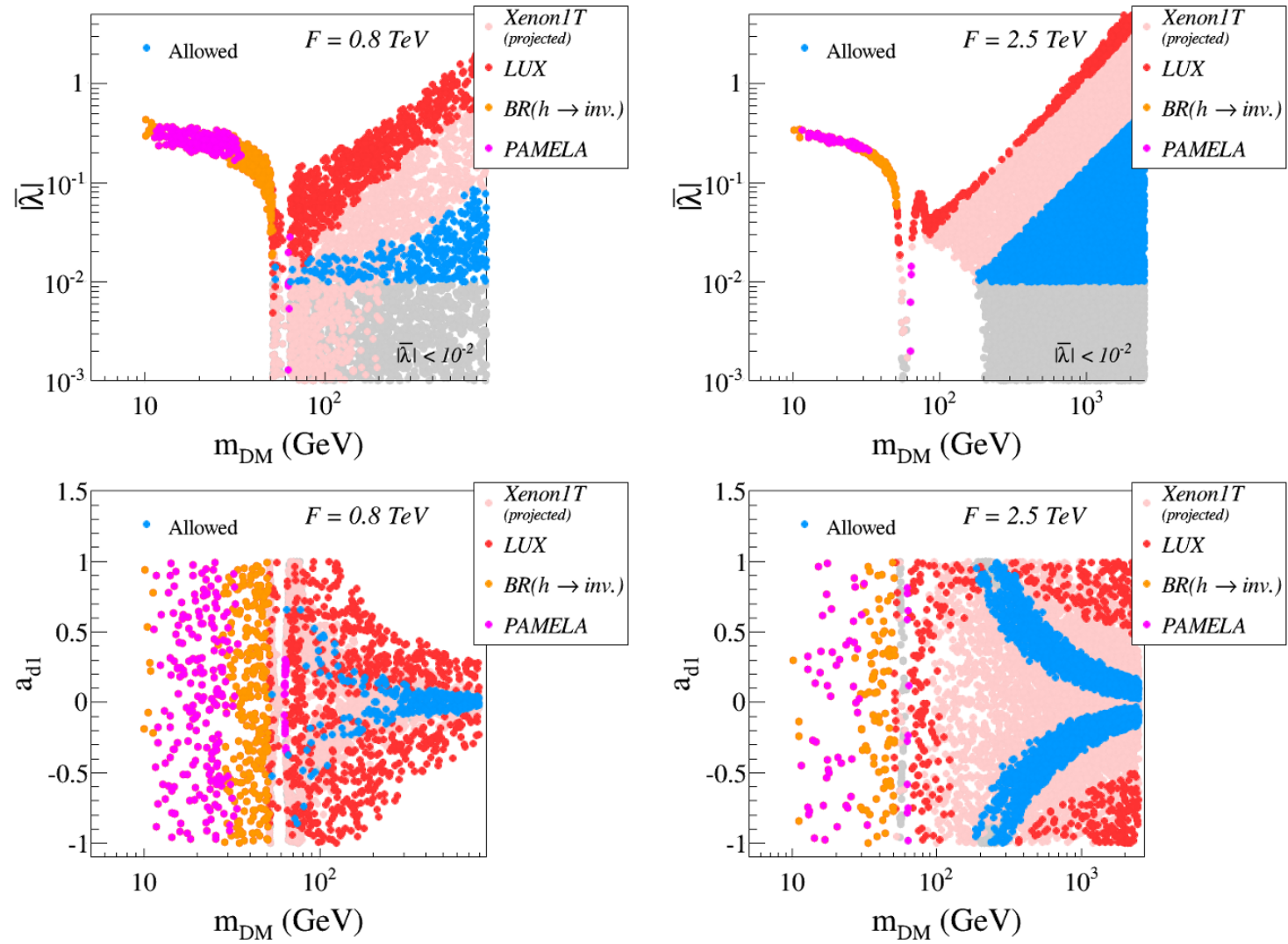

Figure 5. Generic composite singlet $D M$ : experimental constraints in the $|\bar{\lambda}|-m_{\mathrm{DM}}$ (top) and $a_{d 1}-m_{\mathrm{DM}}$ (bottom) planes. We show results for $F=0.8 \mathrm{TeV}$ (left) and $2.5 \mathrm{TeV}$ (right). See text for details.

mostly as Higgs portal DM, being strongly constrained by direct, indirect DM searches and invisible Higgs decays.

We summarize the constraining potential of each of the experiments mentioned at the beginning of section 3 in the planes $\bar{\lambda}-m_{\mathrm{DM}}$ and $a_{d 1}-m_{\mathrm{DM}}$ of figure 5 . Let us first mention that, in some regions of the parameter space, very small values of $\bar{\lambda}$ are necessary to account for the right relic abundance. Although this is typically consistent with most experimental constraints, in the composite framework, both $\lambda_{1}$ (the Higgs self-coupling) and $\bar{\lambda}$ are expected to be generated by the explicit breaking of $\mathcal{G}$ and to be of similar size, i.e. $\mathcal{O}\left(\lambda_{1}\right) \sim \mathcal{O}(|\bar{\lambda}|) \simeq 0.1$. An explicit example can be found in ref. [17], where $\bar{\lambda} \simeq 0.13$ for $\mathcal{G} / \mathcal{H}=\mathrm{SO}(6) / \mathrm{SO}(5)$. Therefore we consider the region with $|\bar{\lambda}|<10^{-2}$ as theoretically disfavored.

In figure 5 , the top (bottom) plots illustrate, in the plane $|\bar{\lambda}|-m_{\mathrm{DM}}\left(a_{d 1}-m_{\mathrm{DM}}\right)$, for $F=0.8$ (left) and $2.5 \mathrm{TeV}$ (right), which regions are currently excluded by direct DM searches (with LUX in red), by indirect DM searches (with PAMELA in magenta) and due to large contributions to the Higgs invisible width (in yellow). The pink regions are currently allowed and will eventually be probed by Xenon1T. The gray regions are experimentally allowed by current data and outside the planned sensitivity of Xenon1T, 
but they are theoretically disfavored. In the bottom plots (for $a_{d_{1}}$ ) most of the gray points are hidden behind the region that will be probed by Xenon1T.

As expected from the discussion above, the low mass region $\left(m_{\mathrm{DM}} \lesssim 100 \mathrm{GeV}\right)$ is almost entirely excluded either by indirect, direct searches or the Higgs invisible width constraints. Let us emphasize that this is valid for both small and large values of $F$. The only points still allowed in the low mass region lie around the Higgs resonance and will be tested by Xenon1T. However most of these points have $|\bar{\lambda}| \lesssim 0.01$, which is difficult to realize in specific models without tuning. Interestingly, for $m_{\mathrm{DM}} \gtrsim 200 \mathrm{GeV}$, a large portion of the parameter space (blue points) with $|\bar{\lambda}| \gtrsim 0.1$ evade all current experimental constraints as well as the projected Xenon1T sensitivity, in contrast with the non-composite Higgs portal scenarios.

Let us emphasize though that the allowed values of $a_{d 1}$ are already highly constrained by LUX and have good prospects to be tested by Xenon1T. Since in a specific model (where $\mathcal{G} / \mathcal{H}$ is specified), $a_{d 1}$ is no longer a free parameter, the allowed region of parameter space will be strongly constrained by direct DM searches. For example, in the case of $\mathcal{G} / \mathcal{H}=\mathrm{SO}(6) \times \mathrm{SO}(5)$ discussed below, $a_{d 1}=1 / 2$ and a large portion of the parameter space is already excluded for low values of $F$. For $F=2.5 \mathrm{TeV}$, if no DM signal is observed in Xenon1T, there will be most probably only a small range of DM masses allowed for a given $a_{d 1}$ value.

\subsubsection{Specific case: $\mathcal{G} / \mathcal{H}=\mathrm{SO}(6) / \mathrm{SO}(5)$}

Although we have taken $a_{d 1}$ as a free parameter, in a specific composite model, $a_{d 1}$ is completely fixed by the choice of the $\operatorname{coset} \mathcal{G} / \mathcal{H}$. Therefore it is instructive to discuss how the general results presented above apply to a specific model. The minimal coset consistent with $\mathcal{H} \subset \mathrm{SO}(4)$ is $\mathcal{G} / \mathcal{H}=\mathrm{SO}(6) / \mathrm{SO}(5)$, where the 5 pNGBs correspond to the Higgs bidoublet and the DM singlet. This case has been discussed in detail in refs. [16, 17], so we limit our discussion to re-interpreting the results obtained previously according to the general parametrization introduced in eq. (3.1).

The $\mathcal{G} / \mathcal{H}=\mathrm{SO}(6) / \mathrm{SO}(5)$ case corresponds to the particular choice of values:

$$
a_{2 H}=a_{d 1}=1 / 2 .
$$

Notice that we keep $c_{4}, d_{4}, \lambda_{H 6}, \lambda_{3}$ and $\lambda_{3}^{\prime}$ as free parameters in our analysis. Once $a_{d 1}$ and $a_{2 H}$ are fixed, we can derive from eq. (3.7) a simple expression for the enhancement (or suppression) of $\sigma_{\mathrm{DMp}}$ with respect to its non-composite value:

$$
\sigma_{\mathrm{DMp}} / \sigma_{\mathrm{DMp}}^{\mathrm{NC}}=(1 \pm r)^{2}, \text { where } r=\frac{2}{\lambda_{\xi h}^{\mathrm{NC}}} \frac{m_{\mathrm{DM}}^{2}}{F^{2}},
$$

where once again $\lambda_{\xi h}^{\mathrm{NC}}$ is the value of the Higgs-DM coupling in the non-composite case required to produce the correct relic abundance. Unlike the general case discussed above, now the enhancement or suppression factor $r$ is fixed (for a given value of $m_{\mathrm{DM}}$ ). Hence, instead of spanning a wide range of values, $\sigma_{\mathrm{DMp}}$ now lies in two possible branches, one for the case $1+r$ (enhancement) and one for $1-r$ (suppression). 

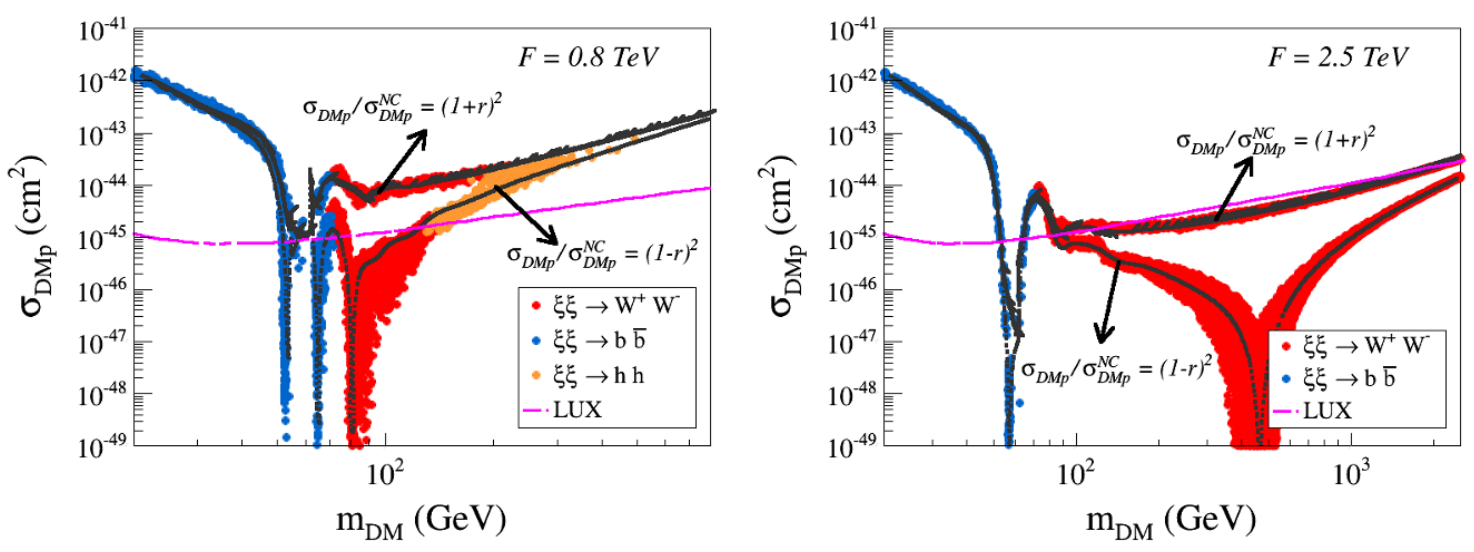

Figure 6. Singlet $D M$ with $\mathcal{G} / \mathcal{H}=\mathrm{SO}(6) / \mathrm{SO}(5)$ : values of the DM-nucleon scattering cross-section as a function of $m_{\mathrm{DM}}$. All points satisfy $0.0941<\Omega_{\mathrm{DM}} h^{2}<0.127$ while the black continuous lines correspond to the approximation of eq. (3.10). We also show with different colors the channels contributing with the largest branching ratio to the effective annihilation cross-section relevant at the time of freeze-out.

In figure 6 , we plot $\sigma_{\mathrm{DMp}}$ as a function of $m_{\mathrm{DM}}$, but now for the particular case of $\mathcal{G} / \mathcal{H}=\mathrm{SO}(6) / \mathrm{SO}(5)$. We also show with black lines the approximation of eq. (3.10) for the enhanced $\left(\sigma_{\mathrm{DMp}}=\sigma_{\mathrm{DMp}}^{\mathrm{NC}}(1+r)^{2}\right)$ and suppressed $\left(\sigma_{\mathrm{DMp}}=\sigma_{\mathrm{DMp}}^{\mathrm{NC}}(1-r)^{2}\right)$ values of the DM-nucleus cross-section. As we can see, the solutions obtained through the random scan follow closely the approximation of eq. (3.10). It is interesting to notice that for $F=0.8 \mathrm{TeV}$ all of the enhanced solutions have already been excluded by LUX, while only the low mass region $\left(m_{\mathrm{DM}} \lesssim 120 \mathrm{GeV}\right)$ of the suppressed branch is allowed. The situation is however quite different for higher values of $F$, where we see that most of the parameter space for both branches has not yet been probed.

Finally we point out that, for $F=0.8 \mathrm{TeV}$, we find no viable solutions for $m_{\mathrm{DM}}>$ $500 \mathrm{GeV}$, even before applying the direct, indirect and Higgs experimental constraints. Once $m_{\mathrm{DM}}>m_{h}$, the annihilation into two Higgses can contribute significantly to $\left\langle\sigma_{\mathrm{ann}} \cdot v\right\rangle$, which can be large even if $\lambda_{\xi h}^{D D} \simeq 0$ due to derivative coupling between DM and Higgs [16]. As a result, the annihilation cross-section becomes too large, suppressing the relic abundance below the WMAP value. However this only happens for small enough values of $F$, as seen in figure 6 .

\subsection{Doublet DM}

If we consider non-minimal cosets, such as e.g. $\mathcal{G} / \mathcal{H}=\mathrm{SO}(6) / \mathrm{SO}(4) \times \mathrm{SO}(2)$ (see section 3.2.3 and e.g. ref. [56]), it is possible to have non-singlet representations for the DM multiplet. Models with multiplet DM differ greatly from the singlet case in two aspects:

- they include tree level (unsuppressed) DM-gauge boson couplings;

- they allow for co-annihilations between the DM multiplet components. 
Since we require invariance under $\mathrm{SO}(4)$, the simplest DM multiplet representation corresponds to a complex doublet. In this case, the non-composite limit $(F \rightarrow \infty)$ corresponds to the Inert Two Higgs Doublet Model or IDM [72].

\subsubsection{Generic composite doublet DM}

The generic doublet case Lagrangian corresponds to eq. (2.4) with the addition of

$$
\begin{aligned}
\mathcal{L}^{(2)}= & \left(D_{\mu} \xi\right)^{\dagger} D^{\mu} \xi-\mu_{\xi}^{2}|\xi|^{2}-\lambda_{3}\left(1+\frac{\lambda_{3}^{\prime}}{F^{2}}|H|^{2}\right)|\xi|^{2}|H|^{2}-\lambda_{4}\left(1+\frac{\lambda_{4}^{\prime}}{F^{2}}|H|^{2}\right)\left|\xi^{\dagger} H\right|^{2} \\
& -\frac{\lambda_{5}}{2}\left(1+\frac{\lambda_{5}^{\prime}}{F^{2}}|H|^{2}\right)\left[\left(\xi^{\dagger} H\right)^{2}+\text { h.c. }\right]+\frac{a_{d 1}}{2 F^{2}} \partial_{\mu}|H|^{2} \partial^{\mu}|\xi|^{2} \\
& +\frac{a_{d 2}}{F^{2}}\left(H^{\dagger} D_{\mu} \xi+\text { h.c. }\right)\left(\xi^{\dagger} D^{\mu} H+\text { h.c. }\right)+\frac{a_{d 3}}{F^{2}}\left[\partial_{\mu}\left(\xi^{\dagger} H+\text { h.c. }\right)\right]^{2} \\
& +\frac{a_{d 4}}{F^{2}}\left[\xi^{\dagger} \overleftrightarrow{D_{\mu}} \xi H^{\dagger} \overleftrightarrow{D}^{\mu} H+\xi^{\dagger} \overleftrightarrow{D_{\mu}} \xi^{C} H^{C \dagger} \overleftrightarrow{D}^{\mu} H-\xi^{\dagger} \vec{\sigma} \overleftrightarrow{D}{ }_{\mu} \xi H^{\dagger} \vec{\sigma} \overleftrightarrow{D}^{\mu} H+\text { h.c. }\right] \\
& -\left[\frac{d_{4}}{F^{2}}|\xi|^{2}\left(y_{t} \bar{Q}_{L} H^{c} t_{R}+y_{b} \bar{Q}_{L} H b_{R}\right)+\text { h.c. }\right] \\
& -\frac{d_{6}}{F^{2}}\left[\xi^{\dagger} \vec{\sigma} \xi\left(y_{t} \bar{Q}_{L} \vec{\sigma} H^{c} t_{R}-y_{b} \bar{Q}_{L} \vec{\sigma} H b_{R}\right)+y_{b} \xi^{c \dagger} \vec{\sigma} \xi \bar{Q}_{L} \vec{\sigma} H^{c} b_{R}+y_{t} \xi^{\dagger} \vec{\sigma} \xi^{c} \bar{Q}_{L} \vec{\sigma} H t_{R}+\text { h.c. }\right],
\end{aligned}
$$

where the coefficients $a_{d 1, \ldots, d 4}, d_{4}, d_{6}, \lambda_{3,4,5}^{\prime}$ are taken to be real $\mathcal{O}(1)$ parameters, the couplings $\lambda_{3,4,5}$ are allowed to vary in the window $[-4 \pi, 4 \pi]$ and $\mu_{\xi}$ is the DM bare mass. The Pauli matrices are denoted by $\vec{\sigma}=\left\{\sigma_{1}, \sigma_{2}, \sigma_{3}\right\}$ and $\phi^{\dagger} \overleftrightarrow{D}_{\mu} \psi \equiv \phi^{\dagger} D_{\mu} \psi-\left(D_{\mu} \psi\right)^{\dagger} \phi$. We also define

$$
\xi=\frac{1}{\sqrt{2}}\left(\begin{array}{c}
-i H^{+} \\
H^{0}+i A^{0}
\end{array}\right),
$$

see section A.2 for more details. After EWSB, the DM multiplet acquires the following masses:

$$
\begin{aligned}
m_{\mathrm{DM}}^{2} \equiv m_{H^{0}}^{2} & =\mu_{\xi}^{2}+\frac{v^{2}}{2}\left(\lambda_{3}+\lambda_{4}+\lambda_{5}+\frac{\lambda_{3} \lambda_{3}^{\prime}+\lambda_{4} \lambda_{4}^{\prime}+\lambda_{5} \lambda_{5}^{\prime}}{2 F^{2}} v^{2}\right), \\
m_{A^{0}}^{2} & =\mu_{\xi}^{2}+\frac{v^{2}}{2}\left(\lambda_{3}+\lambda_{4}-\lambda_{5}+\frac{\lambda_{3} \lambda_{3}^{\prime}+\lambda_{4} \lambda_{4}^{\prime}-\lambda_{5} \lambda_{5}^{\prime}}{2 F^{2}} v^{2}\right), \\
m_{H^{ \pm}}^{2} & =\mu_{\xi}^{2}+\frac{v^{2}}{2} \lambda_{3}\left(1+\frac{\lambda_{3}^{\prime}}{2 F^{2}} v^{2}\right) .
\end{aligned}
$$

In the results presented below we consider only the cases where $m_{H^{0}}<m_{A^{0}}, m_{H^{ \pm}}$, so $H^{0}$ is always the DM. ${ }^{10}$ From eqs. (3.13)-(3.15) we see that the three masses are in principle independent. However, large mass splittings are limited by the perturbativity requirements on the $\lambda_{i}, \lambda_{i}^{\prime}$ couplings.

Although the inclusion of the dimension- 6 operators induced by the strong sector introduces 9 new coefficients, most of the DM properties depend on a subset of these (or appropriate linear combinations). For instance, the Higgs-DM coupling is given by:

$$
\lambda_{\xi h}=\frac{\bar{\lambda}}{2}-\left(a_{d 1}+2 a_{d 2}+4 a_{d 3}\right) \frac{p_{h}^{2}}{4 F^{2}}+a_{d 3} \frac{p_{h}^{2}-2 m_{\mathrm{DM}}^{2}}{F^{2}},
$$

\footnotetext{
${ }^{10}$ However we expect all the results derived below to remain valid in the case $A^{0}$ is the DM particle.
} 
where $p_{h}$ is again the Higgs momentum and

$$
\bar{\lambda} \equiv \sum_{i=3,5} \lambda_{i}\left(1+\lambda_{i}^{\prime} \frac{v^{2}}{F^{2}}\right) .
$$

It is useful to distinguish between the DM-Higgs coupling which enter $s$-channel annihilations $\left(p_{h}^{2} \simeq 4 m_{\mathrm{DM}}^{2}\right)$ and $t$-channel scattering $\left(p_{h}^{2} \simeq 0\right)$. The first case is relevant to $\left\langle\sigma_{\mathrm{ann}} \cdot v\right\rangle$, while the latter enters the calculation of $\sigma_{\mathrm{DMp}}$, hence we define:

$$
\begin{aligned}
\left.\lambda_{\xi h}\right|_{p_{h}^{2}=4 m_{\mathrm{DM}}^{2}} & \equiv \lambda_{\xi h}^{R D}=\frac{\bar{\lambda}}{2}-\left(a_{d 1}+2 a_{d 2}+2 a_{d 3}\right) \frac{m_{\mathrm{DM}}^{2}}{F^{2}}, \\
\left.\lambda_{\xi h}\right|_{p_{h}^{2}=0} & \equiv \lambda_{\xi h}^{D D}=\frac{\bar{\lambda}}{2}-2 a_{d 3} \frac{m_{\mathrm{DM}}^{2}}{F^{2}} .
\end{aligned}
$$

While $\lambda_{\xi h}^{R D}$ is very similar to eq. (3.3) for the singlet case, the coupling relevant for direct detection now contains a contribution from $a_{d 3}$. The latter, however, is only relevant for large DM masses. Also notice that the $H^{0} H^{0} V_{\mu} V^{\mu}$ vertex is not modified by the dimension6 operators driven by the $a_{d 4}$ or $a_{d 2}$ coefficients, since the corresponding operators vanish for $\xi \rightarrow\left(0, H^{0}\right) / \sqrt{2}$ and $H \rightarrow(0, h+v) / \sqrt{2}$.

In the large mass region $\left(m_{\mathrm{DM}} \gg m_{W}\right)$ the annihilation to gauge bosons is open and become the dominant annihilation channel. Even in the pure gauge limit, i.e. when all quartic couplings are set to zero, $\left\langle\sigma_{\mathrm{ann}} \cdot v\right\rangle$ always gets a non-zero contribution from $H^{0} H^{0} \rightarrow W W, Z Z$. Let us focus on the analysis of the latter channel, neglecting the coannihilations, and the $H^{0} H^{0} \rightarrow h h, t t$ channels. The annihilation cross-section into gauge bosons in the high mass region can be approximated by:

$$
\begin{aligned}
\left.\left\langle\sigma_{\mathrm{ann}} \cdot v\right\rangle\right|_{H_{0} H_{0} \rightarrow V V} & \simeq \frac{1}{32 \pi m_{\mathrm{DM}}^{2}}\left(2\left|\mathcal{M}_{T}\right|^{2}+\left|\mathcal{M}_{L}\right|^{2}\right), \text { where } \\
\mathcal{M}_{T}\left(H^{0} H^{0} \rightarrow V_{T} V_{T}\right) & \simeq \frac{g_{V}^{2}}{2}\left(1+\frac{v^{2}}{2 m_{\mathrm{DM}}^{2}} \lambda_{\xi h}^{R D}\right) \simeq \frac{g_{V}^{2}}{2}, \\
\mathcal{M}_{L}\left(H^{0} H^{0} \rightarrow V_{L} V_{L}\right) & \simeq \frac{g_{V}^{2}}{2} \frac{m_{\mathrm{DM}}^{2}}{m_{V}^{2}}\left[\frac{v^{2}}{m_{\mathrm{DM}}^{2}} \lambda_{\xi h}^{R D}+\frac{m_{\mathrm{DM}}^{2}}{\bar{m}_{\xi}^{2}}\left(\frac{\Delta m_{\xi}^{2}}{m_{\mathrm{DM}}^{2}}+2 a_{d 2} \frac{v^{2}}{F^{2}}\right)\right],
\end{aligned}
$$

where $\mathcal{M}_{T}$ and $\mathcal{M}_{L}$ are the contributions to the amplitude from transverse and longitudinal modes respectively and $g_{V}=g\left(g / c_{w}\right), \bar{m}_{\xi}^{2} \equiv\left(m_{\mathrm{DM}}^{2}+m_{H^{+}\left(A^{0}\right)}^{2}\right) / 2, \Delta m_{\xi}^{2} \equiv m_{H^{+}\left(A^{0}\right)}^{2}-$ $m_{\mathrm{DM}}^{2}$, for $V=W(Z)$. From the above expressions we have:

$$
\left\langle\sigma_{\mathrm{ann}} \cdot v\right\rangle>\frac{1}{16 \pi m_{\mathrm{DM}}^{2}}\left|\mathcal{M}_{T}\right|^{2} \simeq \frac{g_{V}^{2}}{32 \pi m_{\mathrm{DM}}^{2}},
$$

resulting in too small relic abundances, unless $m_{\mathrm{DM}} \gtrsim 500 \mathrm{GeV}$. This is a well known result from the IDM $[42,73]$ and it still holds in the composite case, since the contributions from the dimension-6 operators to $\mathcal{M}_{T}$ are negligible. Notice that, in contrast with the noncomposite case [42], for sufficiently large $m_{\mathrm{DM}}$ the annihilation cross-section is driven by the term proportional to $a_{d 2}$. Let us stress though that, as mentioned in section 2 , our effective Lagrangian approach is only valid for $m_{\mathrm{DM}} \lesssim F$. 
From eq. (3.19) we see that if the $\mathcal{M}_{L}$ contribution had to be suppressed one would need small mass splittings among the components of the DM doublet $\left(\Delta m_{\xi}^{2} / \bar{m}_{\mathrm{DM}}^{2} \ll 1\right)$ and the combination

$$
\lambda_{\xi h}^{R D} \frac{v^{2}}{m_{\mathrm{DM}}^{2}}+2 a_{d 2} \frac{v^{2}}{F^{2}}
$$

should be small. Since $\left|\lambda_{\xi h}^{R D}\right|$ can not be made arbitrarily large (otherwise $H^{0} H^{0} \rightarrow h h$ enhances $\left.\left\langle\sigma_{\text {ann }} . v\right\rangle\right)$, we will see below that the above condition limits the possible values of $a_{d 2}$.

\subsubsection{Scan results and experimental constraints}

For the numerical results presented below we scan over all the parameters in eq. (3.11) plus the ones in the Higgs effective Lagrangian (eq. (2.4)) within the following ranges:

$$
\begin{aligned}
2 \mathrm{GeV} & <m_{\mathrm{DM}}<F, \\
m_{\mathrm{DM}} & <m_{A^{0}}<F, \\
\max \left(70 \mathrm{GeV}, m_{\mathrm{DM}}\right) & <m_{H^{ \pm}}<F, \\
-4 \pi & <\bar{\lambda}<4 \pi, \\
10^{-6} & <\lambda_{H 6}<1, \\
-1 & <\lambda_{3}^{\prime}, \lambda_{4}^{\prime}, \lambda_{5}^{\prime}<1, \\
-1 & <c_{4}, a_{2 H}, d_{4}, d_{6}<1, \\
-1 & <a_{d 1}, a_{d 2}, a_{d 3}, a_{d 4}<1 .
\end{aligned}
$$

Again, we scan logarithmically over $\lambda$ 's ranges and linearly on all the other parameters range. Notice that we have conveniently replaced $\lambda_{1}, \lambda_{2}, \lambda_{3}$ and $\mu_{\xi}^{2}$ by $m_{\mathrm{DM}}, m_{A^{0}}, m_{H^{ \pm}}$ and $\bar{\lambda}$. We have also imposed on the scans that viable models have a stable $\mathrm{EW}$ vacuum with $\langle\xi\rangle=0[74]$ and are consistent with LEPII constraints on BSM states, following ref. [75]. We require that new charged scalar particles $\left(H^{ \pm}\right)$have masses larger than $70 \mathrm{GeV}$, see [7678]. Furthermore, as it is well known from IDM results [73], for $m_{\mathrm{DM}} \gtrsim 500 \mathrm{GeV}$ the correct relic abundance can only be obtained for small mass splittings within the DM multiplet. In this mass region we have imposed that $m_{H^{ \pm}}-m_{\mathrm{DM}}<30 \mathrm{GeV}$ and $m_{A^{0}}-m_{\mathrm{DM}}<30 \mathrm{GeV} \cdot{ }^{11}$ We can divide the discussion into two mass regions: a low mass region for $m_{\mathrm{DM}}<m_{h}$ and a high mass region for $m_{\mathrm{DM}}>m_{h}$.

In figure 7, we show the DM-nucleus scattering cross-section for $F=0.8 \mathrm{TeV}$ and $2.5 \mathrm{TeV}$ for $m_{\mathrm{DM}}<m_{h}$ in the top plots. For reference, we also show the corresponding non-composite limit, $F \rightarrow \infty$, in the bottom LH plot. In this region of the parameter space, LEPII $[75,76]$ constraints require a large mass splitting between $H^{0}$ and $A^{0}$ or $H^{ \pm}$. In particular, all the region with $m_{\mathrm{DM}}+m_{A^{0}}<m_{Z}$ is excluded due to the constraints on the $Z$-invisible width. This combined with the lower bound on $m_{H^{ \pm}}$makes co-annihilations negligible for $m_{\mathrm{DM}} \lesssim 60 \mathrm{GeV}$. Since annihilations to gauge bosons are kinematically forbidden in this region, the main features distinguishing the doublet and singlet cases co-annihilations and DM gauge couplings - are negligible for small DM masses and the doublet DM behaves effectively as singlet DM.

\footnotetext{
${ }^{11}$ We have checked that the mass splittings obtained in the scans are always below $35 \mathrm{GeV}$ for all $F$ scales assumed here. For the purpose of this work, the range used in the scans $(<30 \mathrm{GeV})$ is sufficient to discuss the composite DM phenomenology.
} 

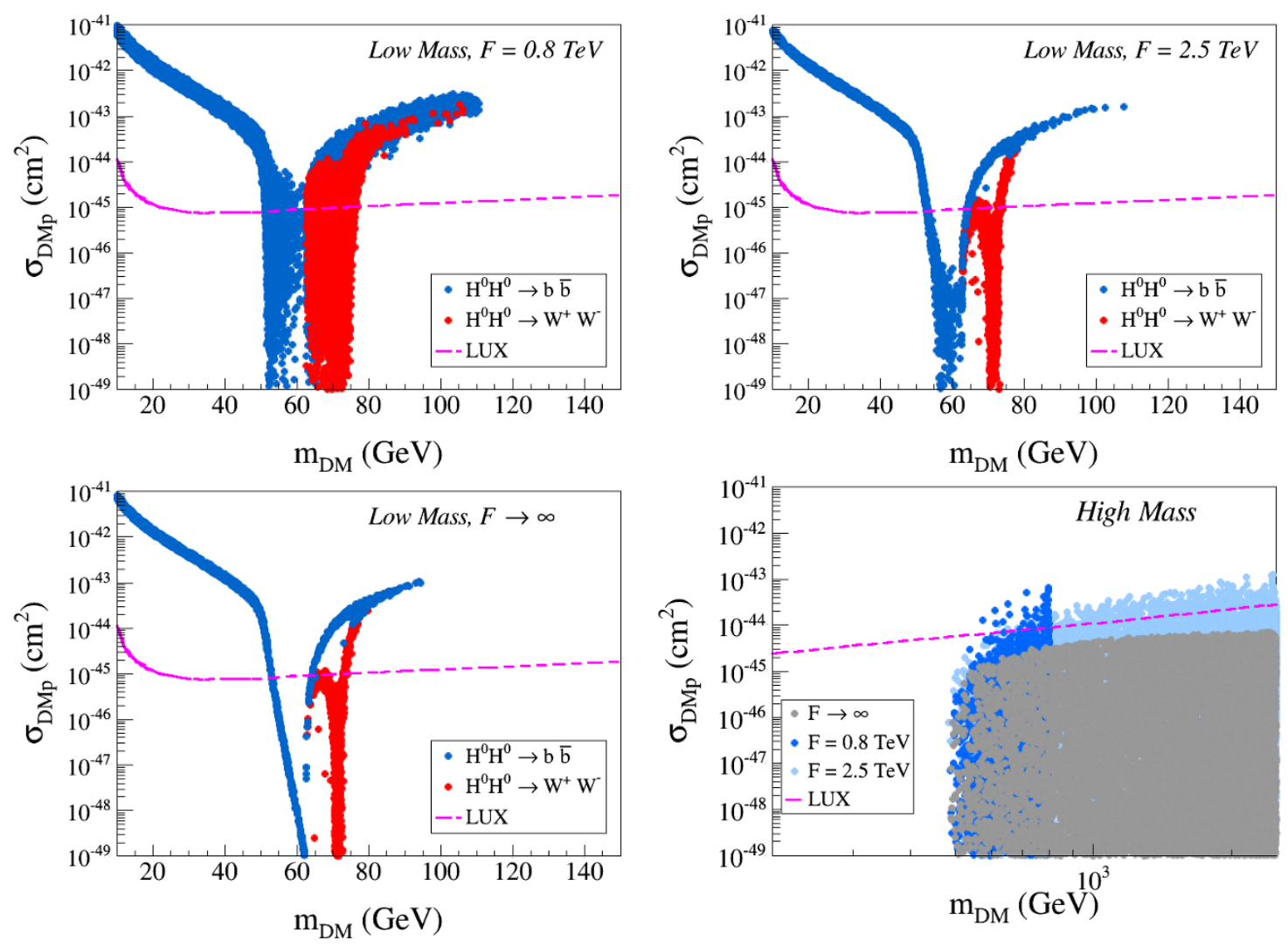

Figure 7. Generic composite doublet DM: values of the DM-nucleon scattering cross-section $\left(\sigma_{\mathrm{DMp}}\right)$ as a function of $m_{\mathrm{DM}}$ satisfying $0.0941<\Omega_{\mathrm{DM}} h^{2}<0.127$. In the top plots $(F=0.8$ and $2.5 \mathrm{TeV})$ and the bottom LH plot $(F \rightarrow \infty)$, we show the low mass region. The channels contributing to the largest branching ratios to the effective annihilation cross-section at freeze out are depicted with different colors. In the bottom RH side plot, we show $\sigma_{\mathrm{DMp}}$ in the high mass region for $F=0.8 \mathrm{TeV}$ (dark blue), $2.5 \mathrm{TeV}$ (light blue) and the non-composite case ( $F \rightarrow \infty$ in gray) superimposed.

Once $m_{\mathrm{DM}} \gtrsim m_{W}$, the $H^{0} H^{0} \rightarrow V V$ process rapidly enhances the DM annihilation cross-section. ${ }^{12}$ For $m_{\mathrm{DM}} \lesssim 110 \mathrm{GeV}$ this enhancement is still partially suppressed by kinematics and can result in the correct relic abundance if there is destructive interference between the $H^{0} H^{0} \rightarrow h^{*} \rightarrow V V$ and $H^{0} H^{0} \rightarrow V V$ amplitudes [79], which happens for $\lambda_{\xi h}^{R D}<0$. For larger values of the DM mass, the contribution from annihilation into gauge bosons is no longer suppressed and generates too small relic abundances, independently of the choice of parameters. This is a well known feature of the IDM and remains valid in the composite scenario. Nonetheless, in the region where $H^{0} H^{0} \rightarrow V V$ is (partially) suppressed, the contributions from the dimension-6 operators to $\lambda_{\xi h}^{R D}$ allow for a wider range of $\bar{\lambda}$ values consistent with $\Omega_{\mathrm{DM}} h^{2} \simeq 0.12$. As a consequence we obtain larger viable parameter space in figure 7 for $F=0.8 \mathrm{TeV}$ and $F=2.5 \mathrm{TeV}$ (top plots), when compared to the non-composite case (bottom LH plot). Also, the allowed low mass region extends to slightly higher values of $m_{\mathrm{DM}}$ due to the presence of the dimension- 6 operators.

\footnotetext{
${ }^{12}$ Since we include the effects of annihilation to off-shell $W^{\prime}$ 's and $Z$ 's $\left(H^{0} H^{0} \rightarrow V^{*} V\right)$, this channel becomes relevant for slightly smaller values of the DM mass $\left(m_{\mathrm{DM}} \gtrsim 60 \mathrm{GeV}\right)$, as seen in figure 7 .
} 

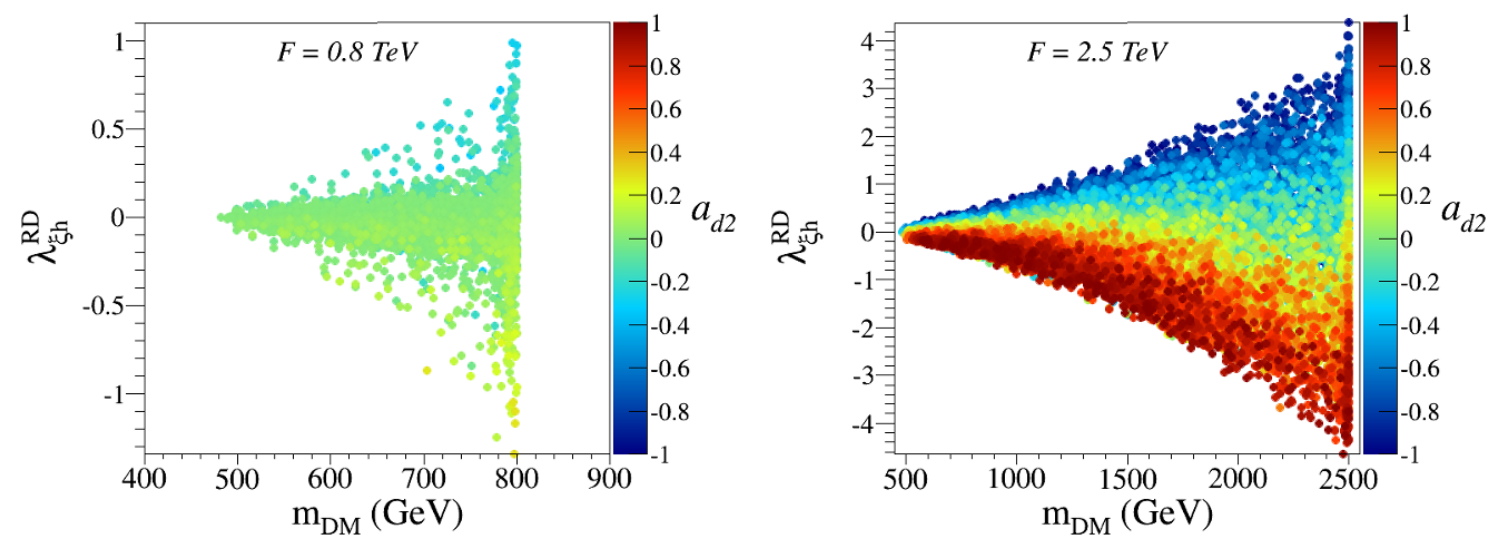

Figure 8. Generic composite doublet DM in the high mass range: values for the dimension-6 coefficient $a_{d 2}$ in the $\lambda_{\xi h}^{R D}-m_{\mathrm{DM}}$ plane for $F=0.8 \mathrm{TeV}$ (left) and $2.5 \mathrm{TeV}$ (right).

In the bottom RH side plot of figure 7 we show values of $\sigma_{\mathrm{DMp}}$ in the high mass region. We see that viable solutions are only obtained for $m_{\mathrm{DM}} \gtrsim 500 \mathrm{GeV}$. The relative mass splittings between the dark components has to be small. Typically we obtain $\Delta m_{\xi}<$ $20 \mathrm{GeV} \ll m_{\mathrm{DM}}$. Since $a_{d 2}$ can take negative values, the allowed mass splittings in the composite case are slightly larger than in the IDM, due to the cancellation between the $a_{d 2}$ and $\Delta m_{\xi}^{2}$ terms in eq. (3.19). In addition, in order to properly quantify the correlations between $\lambda_{\xi h}^{R D}$ and $a_{d 2}$, we show in figure 8 the values of $a_{d 2}$ with gradient colors in the $\lambda_{\xi h}^{R D}-m_{\mathrm{DM}}$ plane for $F=0.8$ and $2.5 \mathrm{TeV}$. We see that $a_{d 2}$ and $\lambda_{\xi h}^{R D}$ typically have opposite signs guaranteeing a suppression of the combination (3.21). We thus see that all the contributions to $\mathcal{M}_{L}$ need to be suppressed in order to account for the right relic abundance. For $F=0.8 \mathrm{TeV}$, all points have $\left|a_{d 2}\right| \lesssim 0.3$, while for $F=2.5 \mathrm{TeV}\left|a_{d 2}\right|$ can be as large as 1 , due to the suppression factor $v^{2} / F^{2} \simeq 0.01$.

From figure 7 we see that $\sigma_{\mathrm{DMp}}$ can take larger values in composite scenarios $(F=0.8$ or $2.5 \mathrm{TeV})$ than in the non-composite case $(F \rightarrow \infty)$. This is particularly visible in the large mass range in the bottom RH side plot where we have $\sigma_{\mathrm{DMp}} \lesssim 10^{-44}\left(10^{-43}\right) \mathrm{cm}^{2}$ for $F \rightarrow \infty(F=0.8 \mathrm{TeV})$. This is easily understood looking at eq. (3.18). In the noncomposite case $\lambda_{\xi h}^{R D}=\lambda_{\xi h}^{D D}=\bar{\lambda} / 2$ and $\sigma_{\mathrm{DMp}} \propto \bar{\lambda}^{2}$, while in composite scenarios we have

$$
\lambda_{\xi h}^{R D}-\lambda_{\xi h}^{D D}=-\left(a_{d 1}+2 a_{d 2}\right) \frac{m_{\mathrm{DM}}^{2}}{F^{2}} .
$$

As a result, $\left|\lambda_{\xi h}^{D D}\right|$, the quartic coupling driving $\sigma_{\mathrm{DMp}}$, can get extra contributions due to the $a_{d i}$ terms in composite models.

Figure 9 illustrates the experimental constraints on the generic composite doublet case, the color code is the same as the one used in section 3.1.3. Once again, points with $|\bar{\lambda}|<0.01$ are theoretically disfavored in realistic composite models, where we expect $\mathcal{O}\left(\lambda_{1}\right) \simeq \mathcal{O}\left(\lambda_{i}\right) \sim 0.1$. Similarly, unless the $a_{d 3}$ and $\bar{\lambda}$ contributions cancel each other in eq. (3.18), at the price of some fine tuning, we will typically have $\mathcal{O}(\bar{\lambda}) \sim \mathcal{O}\left(\lambda_{\xi h}^{D D}\right)$ and too low values of $\lambda_{\xi h}^{D D}$ will also be disfavored. Hence, we consider all points with 

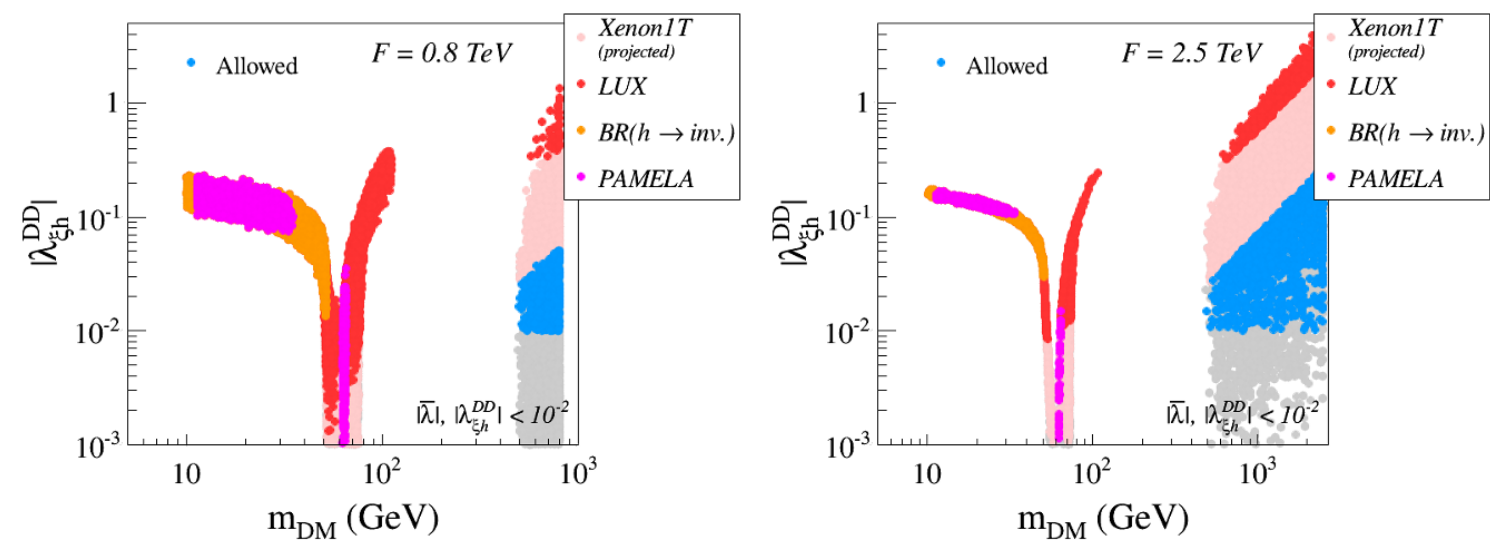

Figure 9. Generic composite doublet DM: experimental constraints in the $\left|\lambda_{\xi h}^{D D}\right|-m_{\text {DM }}$ plane for $F=0.8 \mathrm{TeV}$ (left) and $2.5 \mathrm{TeV}$ (right). See text for details.

$\min \left(\left|\lambda_{\xi \mathrm{h}}^{\mathrm{DD}}\right|,|\bar{\lambda}|\right)<10^{-2}$ as theoretically disfavored and they are shown in gray. As we can see, in the low mass region all points are either excluded by current experimental constraints or involve too small values of $\left|\lambda_{\xi h}^{D D}\right|,|\bar{\lambda}|$ to be theoretically acceptable, except for a small number of points (with $\min \left(\left|\lambda_{\xi \mathrm{h}}^{\mathrm{DD}}\right|,|\bar{\lambda}|\right)>10^{-2}$ ) lying between the resonance region and the $W$ threshold for large values of $F$. Therefore, most of the low mass region of generic composite doublet DM models is already experimentally excluded or theoretically disfavored. In contrast, the high mass region has just been marginally probed by LUX and it even Xenon1T will not be able to test it in its entirety.

\subsubsection{Specific case: $\mathcal{G} / \mathcal{H}=\mathrm{SO}(6) / \mathrm{SO}(4) \times \mathrm{SO}(2)$}

Once again it is interesting to apply the general results presented above to a specific model. Here we take $\mathcal{G} / \mathcal{H}=\mathrm{SO}(6) / \mathrm{SO}(4) \times \mathrm{SO}(2)$, where the only pNGBs are the Higgs bi-doublet and the complex doublet DM. This case corresponds to the effective Lagrangians in eqs. (2.4) and (3.11) with:

$$
a_{2 H}=\frac{1}{2}, a_{d 2}=1 \text { and } a_{d 1}=a_{d 3}=a_{d 4}=0 .
$$

Notice that, according to the results of figure 9, we do not expect many allowed solutions in the low mass range after the LUX constraints have been imposed for $|\bar{\lambda}|>10^{-2}$. Therefore here we only discuss the high mass $\left(m_{\mathrm{DM}}>500 \mathrm{GeV}\right)$ solutions.

One important consequence from specifying the $\mathcal{G} / \mathcal{H}$ coset is that it fixes the value of $a_{d 2}$. As we have seen above, the high mass region requires either large values of $F$ or small values of $a_{d 2}$ (see figure 8 ). Hence, for $\mathcal{G} / \mathcal{H}=\mathrm{SO}(6) / \mathrm{SO}(4) \times \mathrm{SO}(2)$, where $a_{d 2}=1$, we do not expect viable solutions for large DM masses and small values of $F$. This is highlighted in the LH of figure 10 , where we allow $\Omega_{\mathrm{DM}} h^{2}$ to vary outside the $0.09 \lesssim \Omega_{\mathrm{DM}} h^{2} \lesssim 0.12$ range and show the relic density as a function of $m_{\mathrm{DM}}$ for different $F$ values, shown by the color gradient. For this plot, we take $m_{A^{0}}=m_{H^{ \pm}}=m_{\mathrm{DM}}$ and $\bar{\lambda}=\lambda_{i}^{\prime}=0$ since this choice approximately maximizes (minimize) the relic abundance $\left(\left\langle\sigma_{\text {ann }} \cdot v\right\rangle\right)$ and we take $d_{4}=d_{6}=0$, since these coefficients play no major role in the high mass region. 

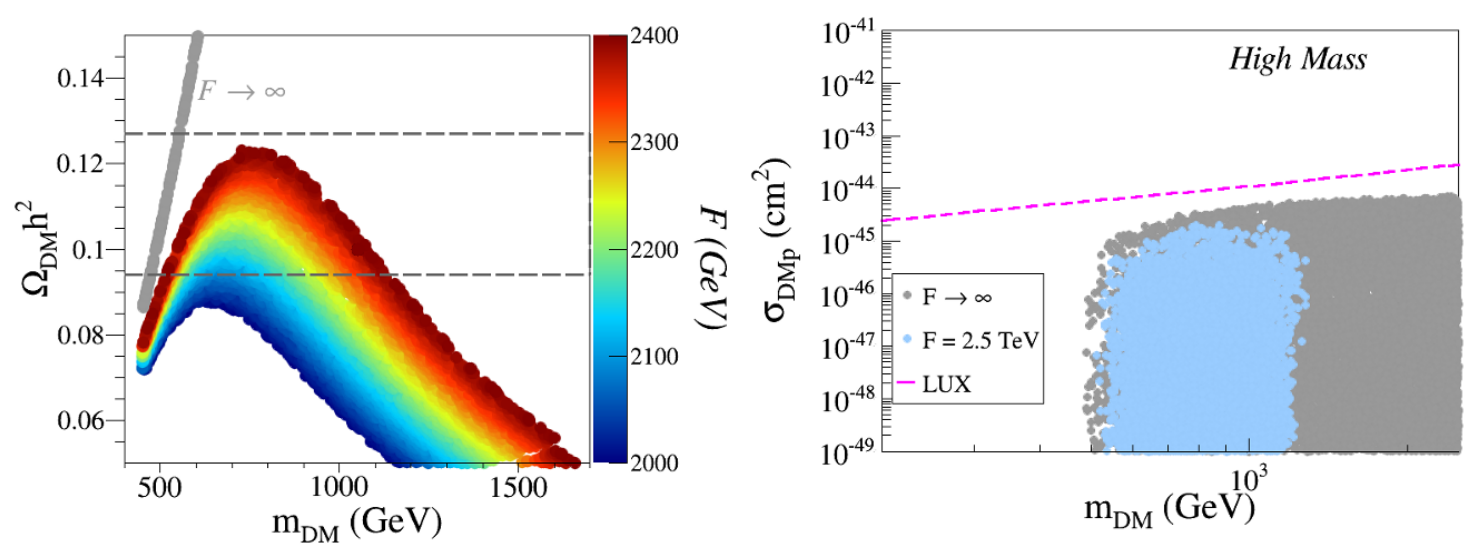

Figure 10. Doublet $D M$ with $\mathcal{G} / \mathcal{H}=\mathrm{SO}(6) / \mathrm{SO}(4) \times \mathrm{SO}(2)$. Left: values of the $\mathrm{DM}$ relic density as a function of $m_{\mathrm{DM}}$ for $2.0 \mathrm{TeV}<F<2.4 \mathrm{TeV}$ fixing $\bar{\lambda}=d_{4}=d_{6}=\lambda_{i}^{\prime}=0$ and $m_{A^{0}}=m_{H^{ \pm}}=m_{\mathrm{DM}}$. The gray points correspond to the non-composite case with $\bar{\lambda}=0$. We also show by dashed lines the $2 \sigma$ CMB bounds on $\Omega_{\mathrm{DM}} h^{2}$. Right: $\sigma_{\mathrm{DMp}}$ as a function of $m_{\mathrm{DM}}$ for points in agreement with the CMB bounds for $F=2.5 \mathrm{TeV}$ and $F \rightarrow \infty$ leaving all quartic and derivative couplings as free parameters.

We can understand the behavior of $\Omega_{\mathrm{DM}} h^{2}$ in the LH of figure 10 by analyzing the $m_{\mathrm{DM}}$ and $F$ dependence of $\left\langle\sigma_{\mathrm{ann}} \cdot v\right\rangle(\xi \xi \rightarrow V V)$ derived in eq. (3.19), but in the "pure gauge limit" (all quartic couplings set to zero). The relic abundance first grows with $m_{\mathrm{DM}}$ due to the usual dependence $\Omega_{\mathrm{DM}} h^{2} \propto 1 /\left\langle\sigma_{\mathrm{ann}} \cdot v\right\rangle \propto m_{\mathrm{DM}}^{2}$. This is indeed what we observe for the lowest range of masses in figure 10 (LH side). The non-composite case in the same limit is plotted for reference with gray points and also show this growing dependence in $m_{\mathrm{DM}}$. When $m_{\mathrm{DM}}$ becomes comparable to $F$, the dimension- 6 derivative operator starts to dominate $\left\langle\sigma_{\mathrm{ann}} \cdot v\right\rangle$ and the contribution from the $a_{d 2}$ term results in $\Omega_{\mathrm{DM}} h^{2} \propto 1 /\left\langle\sigma_{\mathrm{ann}} \cdot v\right\rangle \propto 1 / m_{\mathrm{DM}}^{2}$. For a given $F$ scale there is thus a fixed mass range, bounded from below and above, of viable DM candidates, i.e. in agreement with the CMB bound depicted with vertical dashed gray lines in figure 10 ( $\mathrm{LH}$ side). This viable mass range widen with increasing $F$. Indeed, we see that for e.g. $F=2.4 \mathrm{TeV}$ we have $550 \mathrm{GeV}$ $\lesssim m_{\mathrm{DM}} \lesssim 1.2 \mathrm{TeV}$, while for e.g. $F=2.1 \mathrm{TeV}$ we have $600 \mathrm{GeV} \lesssim m_{\mathrm{DM}} \lesssim 800 \mathrm{GeV}$. In addition, once $F<2 \mathrm{TeV}, \Omega_{\mathrm{DM}} h^{2}<0.09$ for any value of $m_{\mathrm{DM}}$ in the large mass range. Therefore, for viable composite scenarios with $\mathcal{G} / \mathcal{H}=\mathrm{SO}(6) / \mathrm{SO}(4) \times \mathrm{SO}(2)$ one needs to consider $F>2 \mathrm{TeV}$.

In figure 10 ( $\mathrm{RH}$ side) we show results for a scan on the parameter space of $\mathcal{G} / \mathcal{H}=$ $\mathrm{SO}(6) / \mathrm{SO}(4) \times \mathrm{SO}(2)$, where the non-derivative coefficients are still free parameters. We have explicitly verified that even when all non-derivative parameters are allowed to vary there are no viable solutions for $F=0.8 \mathrm{TeV}$. We thus present points only for $F=2.5 \mathrm{TeV}$ and $F \rightarrow \infty$. As shown by the results, for $F=2.5 \mathrm{TeV}$, we only obtain the correct relic abundance for $500 \mathrm{GeV} \lesssim m_{\mathrm{DM}} \lesssim 1.1 \mathrm{TeV}$, as expected from our discussion above.

\subsection{Triplet DM and higher representations}

In sections 3.1 and 3.2 we have discussed the cases of the singlet and complex doublet $\mathrm{DM}$, which can be realized for specific choices of the coset $\mathcal{G} / \mathcal{H}$, such as the $\mathrm{SO}(6) / \mathrm{SO}(5)$ 
and $\mathrm{SO}(6) / \mathrm{SO}(4) \times \mathrm{SO}(2)$ ones discussed above. If one wants to consider even larger symmetry groups $\mathcal{G}$, it is possible that in the low energy effective theory one ends up with a triplet $\mathrm{DM}$ or even higher $\mathrm{SU}(2)_{L}$ representations. As it is well known from noncomposite models $[42,63]$, such cases are highly constrained and tend to require multi-TeV DM masses. In this section we will discuss how these features are affected by the inclusion of the dimension- 6 operators induced by the strong sector. As discussed in section 3 , we focus here on real representations, which correspond to $\xi^{C}=\xi$ and simplify considerably the analysis. Also, after EWSB, the lightest component of the DM candidate from a complex representation usually is a charged field and do not provide a viable DM candidate [42].

The effective DM Lagrangian for any real DM multiplet is given by:

$$
\begin{aligned}
\mathcal{L}^{(2)}= & \left(D_{\mu} \xi\right)^{\dagger} D^{\mu} \xi-\mu_{\xi}^{2}|\xi|^{2}-\lambda_{3}\left(1+\frac{\lambda_{3}^{\prime}}{F^{2}}|H|^{2}\right)|\xi|^{2}|H|^{2} \\
& -\frac{\lambda_{4}}{F^{2}} \xi^{\dagger}\left\{\Gamma^{i}, \Gamma^{j}\right\} \xi H^{\dagger} \sigma^{i} H H^{\dagger} \sigma^{j} H-\frac{\lambda_{5}}{F^{2}} \xi^{\dagger}\left\{\Gamma^{i}, \Gamma^{j}\right\} \xi H^{c \dagger} \sigma^{i} H H^{\dagger} \sigma^{j} H^{c} \\
& +\frac{a_{d 1}}{2 F^{2}} \partial_{\mu}|\xi|^{2} \partial^{\mu}|H|^{2}-\frac{a_{d 4}}{F^{2}} \xi^{\dagger} \vec{\Gamma} \overleftrightarrow{D}^{\mu} \xi H^{\dagger} \vec{\sigma} \overleftrightarrow{D}{ }_{\mu} H \\
& -\frac{d_{4}}{F^{2}}|\xi|^{2}\left(y_{t} \bar{Q}_{L} H^{c} t_{R}+y_{b} \bar{Q}_{L} H b_{R}+\text { h.c. }\right)
\end{aligned}
$$

where the coefficients $a_{d 1, d 4}, d_{4}$ and $\lambda_{3,4,5}^{\prime}$ are taken to be real $\mathcal{O}(1)$ parameters, the couplings $\lambda_{i}$ with $i=3,4,5$ are allowed to vary in the window $[-4 \pi, 4 \pi]$ and $\mu_{\xi}$ is the DM bare mass. The Pauli matrices are again denoted by $\vec{\sigma}=\left\{\sigma_{1}, \sigma_{2}, \sigma_{3}\right\}$ while the $\Gamma$ matrices are the generators of the representation $n$ of $\mathrm{SU}(2)_{L}$ satisfying $\left[\Gamma_{i}, \Gamma_{j}\right]=i \epsilon_{i j k} \Gamma_{k}$ (see appendix A.3 for more details).

\subsubsection{Generic composite triplet DM}

In order to provide some insight on the general behavior of a composite DM multiplet of dimension $n>2$ of $\mathrm{SU}(2)_{L}$ we first focus on the case of the triplet. Later we will discuss how these results generalize to larger representations. In the triplet case, we use the following generators (in the spherical basis):

$$
\Gamma_{1}=\frac{1}{\sqrt{2}}\left(\begin{array}{ccc}
0 & -1 & 0 \\
-1 & 0 & 1 \\
0 & 1 & 0
\end{array}\right), \quad \Gamma_{2}=\frac{1}{\sqrt{2}}\left(\begin{array}{ccc}
0 & i & 0 \\
-i & 0 & -i \\
0 & i & 0
\end{array}\right), \quad \Gamma_{3}=\left(\begin{array}{ccc}
1 & 0 & 0 \\
0 & 0 & 0 \\
0 & 0 & -1
\end{array}\right)
$$

and $\xi=\left(T^{+}, T^{0}, T^{-}\right)^{T}$. Using eq. (3.24) with the above representation for the $\Gamma$ matrices, we obtain the following scalar mass spectrum:

$$
\begin{aligned}
m_{\mathrm{DM}}^{2} \equiv m_{T^{0}}^{2} & =\mu_{\xi}^{2}+v^{2}\left(\frac{1}{2} \lambda_{3}+\frac{1}{4} \lambda_{3} \lambda_{3}^{\prime} \frac{v^{2}}{F^{2}}+\lambda_{5} \frac{v^{2}}{F^{2}}\right) \\
m_{T^{ \pm}}^{2} & =\mu_{\xi}^{2}+v^{2}\left(\frac{1}{2} \lambda_{3}+\frac{1}{4} \lambda_{3} \lambda_{3}^{\prime} \frac{v^{2}}{F^{2}}+\frac{1}{2}\left(\lambda_{4}+\lambda_{5}\right) \frac{v^{2}}{F^{2}}\right) .
\end{aligned}
$$

Notice that in the triplet case, once the values of $m_{\mathrm{DM}}, \lambda_{4}$ and $\lambda_{5}$ are given, the value of $m_{T^{ \pm}}$is fixed.

In the non-composite case $(F \rightarrow \infty)$, the triplet is exactly degenerate (except for small EW loop corrections, see [63]). In the latter case, either LEP constraints excluding the existence of low mass new charged particles or too strong annihilation into gauge (virtual) 
bosons $^{13}$ prevent the existence of viable DM candidate in the low mass range (i.e. for $\left.m_{\mathrm{DM}}<m_{h}\right)$. In the composite scenario, however, this is no longer the case since the dimension- 6 operators induce a mass splitting, given by:

$$
m_{T^{ \pm}}^{2}-m_{\mathrm{DM}}^{2}=\frac{v^{4}}{2 F^{2}}\left(\lambda_{4}-\lambda_{5}\right)
$$

Therefore, assuming $\left|\lambda_{4,5}\right| \lesssim 1$, we can have $m_{\mathrm{DM}}<60 \mathrm{GeV}$ imposing $m_{T^{ \pm}}>70 \mathrm{GeV}$, if $F \lesssim 1.5 \mathrm{TeV}$. Larger values of $F$, however, will not generate sufficient mass splitting to allow for viable solutions in the low mass region. We will analyze this in more details in section 3.3.2.

The effective Higgs-DM coupling in the triplet case is simply:

$$
\begin{aligned}
\lambda_{\xi h} & =\frac{\bar{\lambda}}{2}-a_{d 1} \frac{p_{h}^{2}}{4 F^{2}}, \\
\text { where } \bar{\lambda} & =\lambda_{3}\left(1+\lambda_{3}^{\prime} \frac{v^{2}}{F^{2}}\right)+4 \lambda_{5} \frac{v^{2}}{F^{2}} .
\end{aligned}
$$

Unlike the doublet case, the DM-gauge boson couplings are only affected by one single coefficient $\left(a_{d 4}\right)$ in eq. (3.24). While the contributions to the quartic vertices $\left(\xi^{2} V_{\mu} V^{\mu}\right)$ are usually negligible:

$$
\mathcal{L}_{\text {triplet }} \supset 2 g^{2}\left(1-a_{d 4} \frac{v^{2}}{F^{2}}\right) T^{0} T^{0} W^{+} W^{-},
$$

as they are always suppressed by $v^{2} / F^{2}$, the triple vertex couplings $\left(\xi^{2} V_{\mu}\right)$ can be enhanced at large $m_{\mathrm{DM}}$. In addition let us emphasize that, for the triplet and higher representations, the coupling to the gauge bosons is always larger than in the doublet case because it scales like $\left(n^{2}-1\right)^{2}$. In the case of the doublet, we saw that once $m_{\mathrm{DM}} \simeq m_{W}$, the (co-)annihilation into gauge bosons generate too large $\left\langle\sigma_{\mathrm{ann}} \cdot v\right\rangle$, resulting in too low relic densities until $m_{\mathrm{DM}} \gtrsim 500 \mathrm{GeV}$. This feature is only enhanced in the triplet case, since its couplings to gauge bosons are larger, as it is well known in the non-composite case [42, 63]. We also point out that, due to $C P$-invariance, $Z$ decays to DM pairs are always forbidden, so the $Z$-width constraints do not apply to the case of real triplets.

\subsubsection{Scan results and experimental constraints}

In order to fully cover the allowed parameter space in a model independent approach, we once again scan over the effective Lagrangian couplings within the following ranges:

$$
\begin{aligned}
20 \mathrm{GeV} & <m_{\mathrm{DM}}<F, \\
10^{-6} & <\lambda_{H 6}<1, \\
-4 \pi & <\lambda_{3}<4 \pi, \\
-1 & <\lambda_{3}^{\prime}, \lambda_{4}, \lambda_{5}<1, \\
-1 & <c_{4}, a_{2 H}, d_{4}<1, \\
-1 & <a_{d 1}, a_{d 4}<1 .
\end{aligned}
$$

Again, we scan logarithmically over $\lambda$ 's ranges and linearly on all the other parameters.

\footnotetext{
${ }^{13}$ In the triplet case $T^{0} T^{0} \rightarrow V V^{*}$ is already dominant for $m_{\mathrm{DM}} \gtrsim 50 \mathrm{GeV}$.
} 

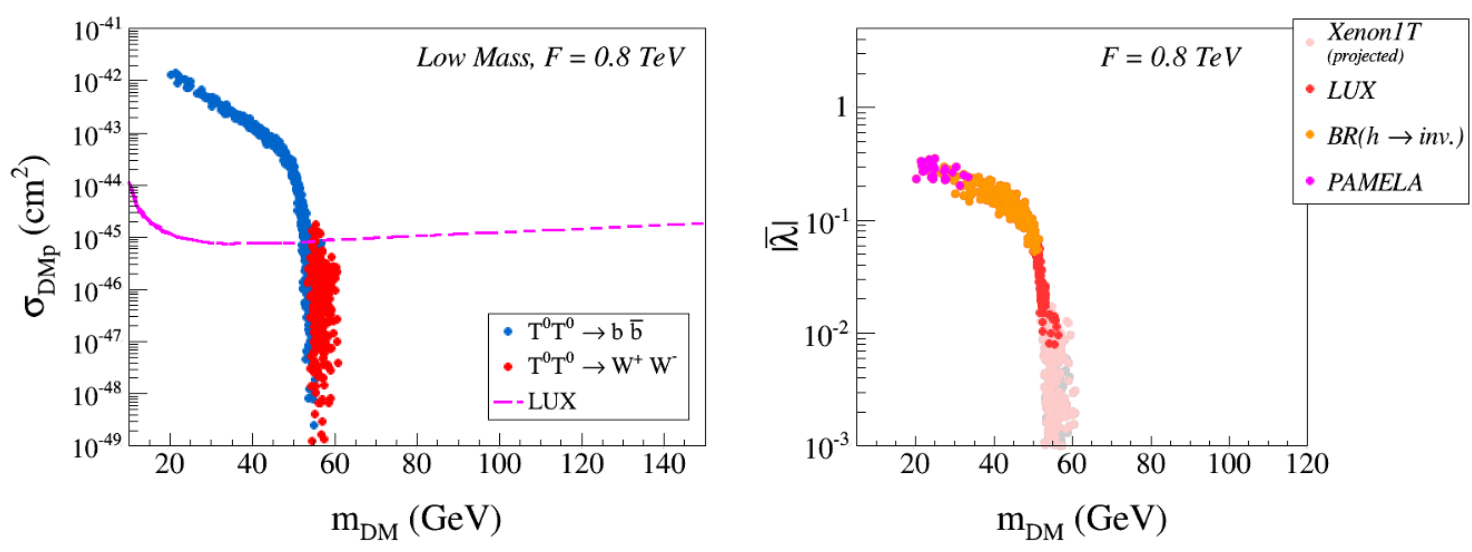

Figure 11. Generic composite triplet DM in the low mass range. Left: values of the DM-nucleon scattering cross-section as a function of $m_{\mathrm{DM}}$ satisfying to $0.0941<\Omega_{\mathrm{DM}} h^{2}<0.127$. The channels contributing with the largest branching ratio to the annihilation cross-section at the time of freezeout are depicted with different colors. Right: experimental constraints on the low mass region. See text for details.

The results of these scans for the low mass region are shown in the LH side of figure 11, where we show the mass splitting and the DM-nucleus scattering cross-section as a function of $m_{\mathrm{DM}}$. We take $F=0.8 \mathrm{TeV}$, since higher values of $F$ will only reduce the allowed region, due to the suppression of the mass splitting between the neutral and the charged component. We also show by blue (red) points the region where $T^{0} T^{0} \rightarrow \bar{b} b\left(T^{0} T^{0} \rightarrow\right.$ $W^{(*)} W$ ) dominates the annihilation cross-section in the early universe. We see that, for $m_{\mathrm{DM}} \simeq 50 \mathrm{GeV}$, annihilations to (off-shell) gauge bosons become dominant and, for $m_{\mathrm{DM}} \gtrsim$ $60 \mathrm{GeV},\left\langle\sigma_{\text {ann }} \cdot v\right\rangle$ becomes too large, resulting in relic abundances below the CMB bounds. In the RH side of figure 11 we show the experimental constraints on the low mass region. As we can see, most of the parameter space is excluded by experimental constraints, or theoretically disfavored for extremely low values of $\bar{\lambda}\left(<10^{-2}\right)$. Only a small number of points within Higgs-resonance and $W$ threshold and with $\bar{\lambda}>10^{-2}$ are still viable and will eventually be tested by Xenon1T experiment. We conclude that, despite allowing for large mass splittings, the composite triplet scenario, alike the non-composite case, remains mostly excluded in the low mass region.

In a way analogous to the doublet scenario, for $m_{\mathrm{DM}} \gg m_{W}$, the annihilation crosssection into gauge bosons becomes suppressed enough to give rise to the right relic abundance. In this high mass region, it takes the following form:

$$
\begin{aligned}
\left.\left\langle\sigma_{\mathrm{ann}} \cdot v\right\rangle\right|_{T_{0} T_{0} \rightarrow V V} & \simeq \frac{1}{32 \pi m_{\mathrm{DM}}^{2}}\left(2\left|\mathcal{M}_{T}\right|^{2}+\left|\mathcal{M}_{L}\right|^{2}\right), \text { where } \\
\mathcal{M}_{T}\left(T^{0} T^{0} \rightarrow V_{T} V_{T}\right) & \simeq 2 g_{V}^{2}\left[1-a_{d 4} \frac{v^{2}}{F^{2}}+\frac{\bar{\lambda}}{8} \frac{v^{2}}{m_{\mathrm{DM}}^{2}}-a_{d 1} \frac{v^{2}}{2 F^{2}} m_{\mathrm{DM}}^{2}\right] \\
& \simeq 2 g_{V}^{2}\left(1-a_{d 4} \frac{v^{2}}{F^{2}}+\frac{v^{2}}{2 m_{\mathrm{DM}}^{2}} \lambda_{\xi h}\right), \\
\mathcal{M}_{L}\left(T^{0} T^{0} \rightarrow V_{L} V_{L}\right) & \simeq \frac{g_{V}^{2}}{2} \frac{v^{2}}{m_{V}^{2}} \lambda_{\xi h} \simeq \lambda_{\xi h},
\end{aligned}
$$



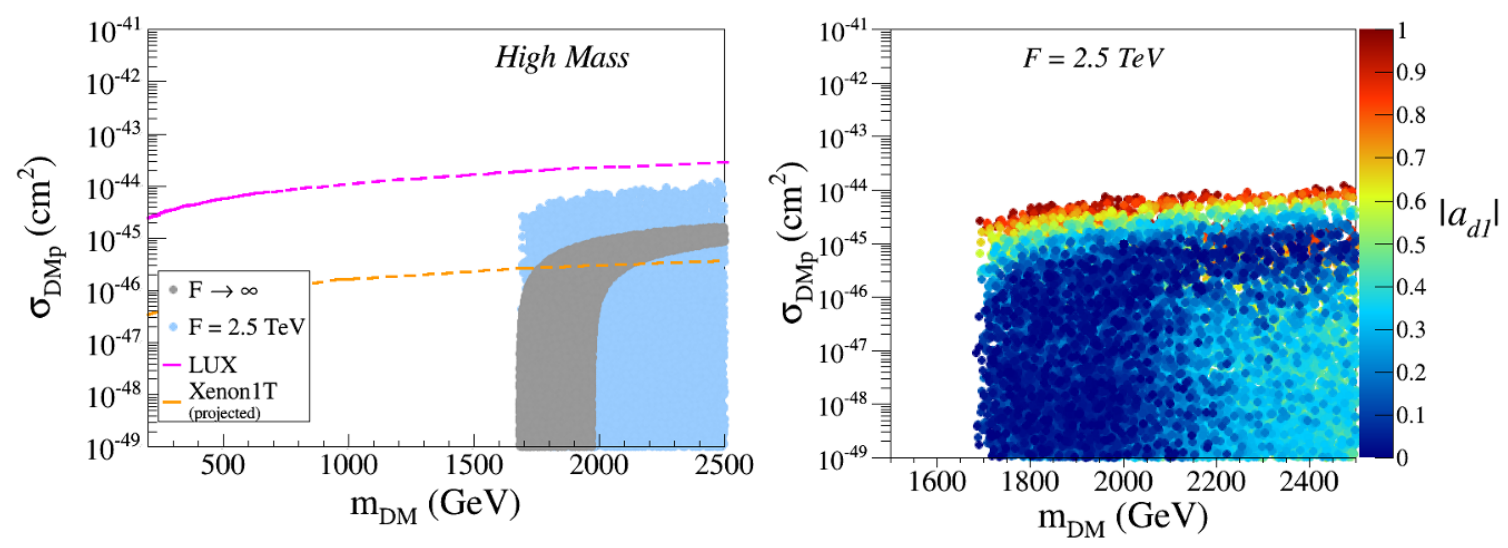

Figure 12. Generic composite triplet DM in the large mass range: values of the DM-nucleon scattering cross-section as a function of $m_{\mathrm{DM}}$ satisfying to $0.0941<\Omega_{\mathrm{DM}} h^{2}<0.127$. Right: values of $\left|a_{d 1}\right|$ in the $\sigma_{\mathrm{DMp}}-m_{\mathrm{DM}}$ plane for $F=2.5 \mathrm{TeV}$.

where we have taken $m_{\mathrm{DM}} \simeq m_{T^{+}}$, since the small mass splitting can be neglected in the high mass region. Notice that in the large mass regime, we typically have $\lambda_{\xi h} v^{2} /\left(2 m_{\mathrm{DM}}^{2}\right) \ll$ 1 , and for the $F \gg v$ considered here we also have $a_{d 4} v^{2} / F^{2}<1$. These two contributions to $\mathcal{M}_{T}$ will thus typically be small.

In the LH side of figure 12 we show the high mass region results for $F=2.5 \mathrm{TeV}$ and the non-composite case $(F \rightarrow \infty)$. As we can see, the lowest $m_{\mathrm{DM}}$ values which generate the correct relic abundance are $m_{\mathrm{DM}} \gtrsim 1.7 \mathrm{TeV}$. Hence there are no possible solutions for $F=0.8 \mathrm{TeV}$, since our effective Lagrangian assumes $m_{\mathrm{DM}}<F$. We also show the LUX and projected Xenon1T constraints. As we can see, the large mass region has not yet been probed by LUX and will only be partially tested by Xenon1T. From this plot, we see that while in the non-composite case a non-observation of signal in Xenon1T would imply $m_{\mathrm{DM}} \lesssim 2.1 \mathrm{TeV}$, in the composite scenario this upper bound can be easily avoided due to the presence of the dimension- 6 derivative operator.

Let us emphasize that for such large triplet masses the Sommerfeld effect can be nonnegligible [80]. A full treatment of the Sommerfeld enhancement within composite dark matter scenarios is however beyond the scope of this work. Let us mention though that, already in the non-composite case, one expects a non-perturbative enhancement factor of the effective coupling driving the annihilation cross section which is approximately constant away from the resonances and is about 1.6 in the triplet case $[42,80]$. This implies an even stronger lower bounds on $m_{\mathrm{DM}}$ in the high mass region. For instance, if the Sommerfeld enhancement [81] is included in the non-composite limit, one would obtain $m_{\mathrm{DM}} \gtrsim 2.1 \mathrm{TeV}$, instead of $1.7 \mathrm{TeV}$.

The effective Lagrangian in the non-composite limit contains only two parameters $\left(\lambda_{3}\right.$ and $m_{\mathrm{DM}}$ ) and, as in the singlet case, the DM-nucleus cross-section value is fixed (for a given $m_{\mathrm{DM}}$ ) once the constraint on the relic abundance is imposed. The band seen in the LH side of figure 12 for $F \rightarrow \infty$ is simply due to the fact that we allow $\Omega_{\mathrm{DM}} h^{2}$ to vary within the interval $[0.0941,0.127]$. For the composite case, however, $\lambda_{\xi h}$ receives contributions 
from the dimension- 6 coefficients $\lambda_{3}^{\prime}$ and $a_{d 1}$. In this case, we can have larger or smaller values of $\sigma_{\mathrm{DMp}}$ (when compared to the non-composite case), depending on the values of $a_{d 1}$. In the RH side of figure 12 we show the values of $\left|a_{d 1}\right|$ in the $\sigma_{\mathrm{DMp}}-m_{\mathrm{DM}}$ plane. We can clearly see that values of $\left|a_{d 1}\right| \gtrsim 0.3$ correspond to the regions where the DM-nucleus cross-section is either suppressed or enhanced with respect to the non-composite values. It is also interesting to notice that, while the low mass region requires $F \lesssim 1.2 \mathrm{TeV}$ in order to generate enough mass splitting, the high mass region requires $F>m_{\mathrm{DM}} \gtrsim 1.7 \mathrm{TeV}$. Hence there are no values of $F$ for which there are solutions in both the low mass and the high mass regions. Furthermore, since the low mass region is already excluded by LUX, the triplet case can only provide a DM candidate for $F>1.7 \mathrm{TeV}$.

\subsubsection{Higher representations}

Since the effective Lagrangian in eq. (3.24) is valid for any real DM representation, we can readily extrapolate the general conclusions obtained for the triplet case to higher dimensional representations. Since only odd representations contain a electrically neutral component, the possible representations higher than the triplet are the quintuplet $(n=5)$, the septuplet $(n=7)$ [63]. For any $n$-odd representation a mass splitting between the neutral (DM) and charged components is always induced by the dimension- 6 operators proportional to $\lambda_{4,5}$ in composite scenarios. We expect the allowed solutions in the low mass region to be similar to the triplet case shown in figure 11. Consequently, in the case of higher representations, most of the low mass region is expected to be excluded by experimental constraints or theoretically disfavored.

For the high mass range, given the results obtained for the doublet and triplet DM, one can expect that the threshold mass is the same in composite and non-composite scenarios. In the latter case, one can check that the total effective annihilation cross-section in the early universe has a given dependence in $n[42,63]$. In the pure gauge limit it scales as $\left(n^{2}-1\right)\left(n^{2}-3\right) / n$ for a multiplet of dimension $n$ so that for $n=5(7)$ one would have $m_{\mathrm{DM}}^{\min } \simeq 4.3(7.5) \mathrm{TeV}$. Again, one expect that Sommerfeld corrections will push these thresholds to even higher masses, see [80] in the non-composite limit.

If we consider compositeness as a solution to the little hierarchy problem, we must require $F \lesssim 3 \mathrm{TeV}$ in order to obtain a fine-tuning of the order of $1 \%$ or lower [39]. Therefore, since $m_{\mathrm{DM}}<F$, we see that the triplet is the highest DM representation allowed. Although there is still a small range of low DM masses allowed for all representations $\left(50 \mathrm{GeV} \lesssim m_{\mathrm{DM}} \lesssim 60 \mathrm{GeV}\right)$, these solutions usually require extremely small values of $\lambda_{\xi h}$, which are unlikely to be generated in realistic models, where one expects $\lambda_{\xi h} \sim \mathcal{O}(0.01-1)$. Consequently we conclude that Dark Matter representations higher than the triplet are highly disfavored in composite DM models.

\section{Conclusions}

The nature of DM is one of the greatest conundrums of our time. In spite of the fact that DM constitutes $85 \%$ of the total matter in the Universe, it continues to evade direct experimental observation. A reason for that may be connected to the fact that DM may 
be not elementary. Having this in mind we investigate composite multiplets that can arise in composite Higgs models and study the conditions under which they can be a suitable DM candidate.

We considered a class of composite models where the only composite states present in the low energy effective theory are the Higgs and the DM, the first being a bi-doublet and the latter a multiplet of $\mathrm{SO}(4)$, both pNGBs of a spontaneous broken global symmetry of a new unknown strongly coupled sector. We constructed and parametrized the most general effective Lagrangian up to dimension-6 operators under these general assumptions. We then checked if the different DM SO(4) multiplet candidates could account for all the DM assuming that the freeze-out mechanism is driving the relic abundance. For different DM representations under $\mathrm{SU}(2)_{L}$ (or $\mathrm{SO}(4)$ ) we derived the main DM observables (relic abundance, spin-independent DM-nucleon scattering cross-section, annihilation cross-sections) and imposed experimental constraints from direct and indirect detection experiments as well as LHC constraints from invisible Higgs decays.

In the generic singlet DM scenario (arbitrary cosets), the DM-nucleon scattering crosssection can be significantly suppressed and can evade all current experimental bounds for $m_{\mathrm{DM}} \gtrsim 100 \mathrm{GeV}$ (except for a small discontinuity near the Higgs resonance), if $F \gtrsim$ $0.8 \mathrm{TeV}$. However, once a specific model is considered (with a given coset), it can be severely constrained by the DM observables. As an example, we discussed the case $\mathcal{G} / \mathcal{H}=$ $\mathrm{SO}(6) / \mathrm{SO}(5)$.

The composite doublet DM models differ from the singlet one due to tree level DMgauge boson couplings and co-annihilations between DM multiplet components. There are two viable mass regions, one below the Higgs mass and one above $\sim 500 \mathrm{GeV}$. Most of the points in the low mass region are either excluded by data or theoretically disfavored. For $m_{\mathrm{DM}} \gtrsim 500 \mathrm{GeV}$, the annihilation into gauge bosons get suppressed enough to account for the DM relic abundance, just as in the IDM scenario. In the case $\mathcal{G} / \mathcal{H}=\mathrm{SO}(6) / \mathrm{SO}(4) \times$ $\mathrm{SO}(2)$ presented in section 3.2.3, the dimension-6 operators allow for solutions only if $F>$ $2 \mathrm{TeV}$. The DM mass is then constrained to the range $600(550) \mathrm{GeV} \lesssim m_{\mathrm{DM}} \lesssim 0.8(1.2) \mathrm{TeV}$ for $F=2.1(2.4) \mathrm{TeV}$.

The composite triplet DM scenario is also mostly excluded by data or theoretical considerations in the low mass region, despite allowing for larger mass splittings between neutral and charged components than in the non-composite scenarios. The large mass region will only be partially tested by Xenon1T. We also mention that while in the noncomposite triplet scenario a non-observation of a signal in Xenon1T would provide an upper bound on $m_{\mathrm{DM}}$, in the composite case this upper bound could be easily avoided. Finally, we have also examined higher representations and concluded they are highly disfavored in composite Higgs models if one requires small fine-tuning in the $\mathrm{EW}$ sector (or $F<3 \mathrm{TeV}$ ).

We have shown that DM in various representations in composite models can reproduce the correct relic abundance and still be compatible with limits on the Higgs invisible width and from the non-observation of DM in direct and indirect detection experiments. These models will be further put to the test by future LHC, direct and indirect detection data, either excluding them completely or revealing some exciting new physics. 


\section{Acknowledgments}

We are grateful to Gabriele Ferretti and Eduardo Ponton for useful discussions. This work was supported by Fundação de Amparo à Pesquisa do Estado de São Paulo (FAPESP) and Conselho Nacional de Ciência e Tecnologia ( $\mathrm{CNPq})$. This work was also supported by the U.S. Department of Energy (DOE) under grant Contract Number DE-SC00012567. R.Z.F. thanks the Kavli Institute for Theoretical Physics in UC Santa Barbara for its hospitality, where part of this work was completed. This research was also supported in part by the National Science Foundation under Grant No. NSF PHY11-25915 and by the European Union FP7 ITN INVISIBLES (Marie Curie Actions, PITN-GA-2011-289442). A.L. thanks HEPHY Vienna for its hospitality, where part of this work was completed. LLH is supported through an "FWO-Vlaanderen" post doctoral fellowship project number 1271513. LLH also recognizes partial support from the Strategic Research Program "High Energy Physics" of the Vrije Universiteit Brussel and from the Belgian Federal Science Policy through the Interuniversity Attraction Pole P7/37 "Fundamental Interactions".

\section{A Dimension six operators for composite DM}

In order to compute the DM observables relevant for our results in section 3, it is essential to identify the relevant operators induced by the new strong sector. In sections 3.1, 3.2 and 3.3 we presented the effective Lagrangians considered in our analyses. Here we will derive them in detail and justify (when necessary) our choice of operators. First we consider the SM dimension-6 operators. In addition to the ones listed in $\mathcal{L}_{6}$ (eq. (2.4)) there are other SM dimension-6 operators that are in agreement with the assumptions discussed in section 2 but were not considered in our analysis. Assuming minimally-coupled theories and a specific basis, the following dimension- 6 operators can be induced at tree-level by integrating out heavy states with spin $\leqslant 1[48-53]$ :

$$
\begin{aligned}
& \frac{c_{H Q 1} i}{F^{2}}\left(H^{\dagger} \sigma^{j} \overleftrightarrow{D^{\mu}} H\right)\left(\bar{Q}_{L} \sigma^{j} \gamma_{\mu} Q_{L}\right), \quad \frac{c_{H Q 2} i}{F^{2}}\left(H^{\dagger} \overleftrightarrow{D}^{\mu} H\right)\left(\bar{Q}_{L} \gamma_{\mu} Q_{L}\right), \\
& \frac{c_{H u} i}{F^{2}}\left(H^{\dagger} \overleftrightarrow{D}^{\mu} H\right)\left(\bar{u}_{R} \gamma_{\mu} u_{R}\right), \quad \frac{c_{H d} i}{F^{2}}\left(H^{\dagger} \overleftrightarrow{D}^{\mu} H\right)\left(\bar{d}_{R} \gamma_{\mu} d_{R}\right), \quad \frac{c_{H u d} i}{F^{2}}\left(H^{c \dagger} \overleftrightarrow{D}^{\mu} H\right)\left(\bar{u}_{R} \gamma_{\mu} d_{R}\right) \\
& \frac{a_{W} i g}{M_{\rho}^{2}}\left(H^{\dagger} \sigma^{j} \overleftrightarrow{D^{\mu}} H\right) D^{\nu} W_{\mu \nu}^{j}, \\
& \left.\frac{a_{2 W} g^{2}}{M_{\rho}^{2} g_{\rho}^{2}}\left(D^{\mu} W_{\mu \nu}\right)^{j}\left(D_{\beta} W^{\beta \nu}\right)^{j}, \quad \frac{a_{2 B} g^{\prime 2}}{M_{\rho}^{2} g_{\rho}^{2}}\left(\partial^{\mu} B_{\mu \nu}\right)\left(\partial_{\beta} B^{\beta \nu}\right), \quad \frac{a_{2 G} g_{s}^{2}}{M_{\rho}^{2} g_{\rho}^{2}}\left(D^{\mu} G_{\mu \nu}\right)^{a}\left(D_{\beta} G^{\beta \nu}\right)^{a}, \text { (A. } 2\right)
\end{aligned}
$$

where $H^{\dagger} \sigma^{i} \overleftrightarrow{D}^{\mu} H \equiv H^{\dagger} \sigma^{i} D^{\mu} H-\left(D^{\mu} H\right)^{\dagger} \sigma^{i} H, M_{\rho} \simeq g_{\rho} F$, and $g_{\rho}$ is the typical coupling of the Higgs doublet and SM fermions to the heavy resonances $\left(g_{\rho} \lesssim 4 \pi\right)$. The coefficients $a_{i}$ and $c_{i}$ are $\mathcal{O}(1)$ numbers. The operators in (A.3), (A.4) have at least a suppression of $1 / g_{\rho}^{2}$ compared with the operators in $\mathcal{L}_{6} \cdot{ }^{14}$ Additionally, the operator proportional to $a_{W}$ (A.3)

\footnotetext{
${ }^{14}$ Note that the suppression of the operators in (A.3), (A.4) compared with $\mathcal{L}_{6}$ is larger as the theory is close to the strong-coupling limit, i.e. when the typical coupling is $g_{\rho} \sim 4 \pi$.
} 
contributes at tree-level to the $S$ parameter, which is very constrained by the electroweak precision data [40]. For these reasons, we do not consider these operators in our effective Lagrangians. On the other hand, (A.1), (A.2) have the same suppression factor $\left(1 / F^{2}\right)$ as the operators in $\mathcal{L}_{6}$ and should, in principle, be considered. However, these operators modify the gauge couplings to fermions which are in great agreement with the SM [40]. We can try to avoid this constraint by absorbing the universal part of the vertex corrections to a redefinition of the electroweak gauge fields $[82,83]$. This restores the gauge couplings to their SM values, but it generates contributions to the electroweak precision parameters, which also are severely constrained by the experimental data. Therefore, all the operators in (A.1), (A.2) are extremely constrained and we disregard them. We also neglect the 4 -fermion operators since they do not affect the DM observables discussed here. Finally, operators that can only be generated at one-loop level, such as $\bar{Q}_{L} \sigma^{j} \sigma^{\mu \nu} \psi_{R} H^{c} W_{\mu \nu}^{j}$, are suppressed with respect to the operators in $\mathcal{L}_{6}$ and can also be neglected.

In the remaining sections we compute the $\xi$-dependent part of the effective DM Lagrangian $\left(\mathcal{L}^{(2)}\right)$ for specific DM representations. In order to be consistent and also to reduce the number of free parameters, we assume in the DM sector the same simplifications previously required in the Higgs sector, i.e.

- we neglect the suppressed operators;

- from the electroweak precision data [40] we know that the $c$ 's in (A.1), (A.2) must be very small $\left(c_{H \psi} \ll 1\right)$. Here we assume that the analogous coefficients in the DM sector are also suppressed.

\section{A.1 Singlet DM}

The case of singlet DM is trivial since $\xi$ can only couple to operators which are singlet under $\mathrm{SU}(2)_{L} \times \mathrm{U}(1)_{Y}$. The only operators (up to dimension 6) containing two DM fields and the Higgs field contributing to the scalar potential are given by

$$
\xi^{2}, \xi^{2}|H|^{2}, \xi^{2}|H|^{4}
$$

The dimension- 6 derivative interactions, corresponding to the effective CCWZ, must preserve the symmetry $\mathrm{SO}(4) \subset \mathcal{H}$, hence the only allowed operators are

$$
\xi \partial_{\mu} \xi \partial^{\mu}|H|^{2},|H|^{2} \partial_{\mu} \xi \partial^{\mu} \xi, \xi^{2}\left(D_{\mu} H\right)^{\dagger} D^{\mu} H .
$$

The last two operators can be eliminated after the following field redefinitions

$$
H \rightarrow H+\frac{a}{4 F^{2}} \xi^{2} H, \quad \xi \rightarrow \xi+\frac{a^{\prime}}{F^{2}}|H|^{2} \xi,
$$

then,

$$
\begin{aligned}
\left(D_{\mu} H\right)^{\dagger} D^{\mu} H+\frac{1}{2} \partial_{\mu} \xi \partial^{\mu} \xi \rightarrow & \left(D_{\mu} H\right)^{\dagger} D^{\mu} H+\frac{1}{2} \partial_{\mu} \xi \partial^{\mu} \xi+\frac{a+a^{\prime}}{2 F^{2}} \xi \partial_{\mu} \xi \partial^{\mu}|H|^{2} \\
& +\frac{a}{F^{2}} \xi^{2}\left(D_{\mu} H\right)^{\dagger} D_{\mu} H+\frac{a^{\prime}}{F^{2}}|H|^{2} \partial_{\mu} \xi \partial^{\mu} \xi+\mathcal{O}\left(\frac{1}{F^{4}}\right)
\end{aligned}
$$


From the above result we see that choosing the coefficients $a$ and $a^{\prime}$ properly, the operators $|H|^{2} \partial_{\mu} \xi \partial^{\mu} \xi$ and $\xi^{2}\left(D_{\mu} H\right)^{\dagger} D^{\mu} H$ can always be eliminated. Therefore, we obtain the following effective Lagrangian for the singlet DM field

$$
\begin{aligned}
\mathcal{L}^{(2)}= & \frac{1}{2} \partial_{\mu} \xi \partial^{\mu} \xi-\frac{1}{2} \mu_{\xi}^{2} \xi^{2}-\frac{\lambda_{3}}{2}\left(1+\frac{\lambda_{3}^{\prime}}{F^{2}}|H|^{2}\right) \xi^{2}|H|^{2}+\frac{a_{d 1}}{F^{2}} \partial_{\mu} \xi^{2} \partial^{\mu}|H|^{2} \\
& -\frac{1}{2} \frac{d_{4}}{F^{2}} \xi^{2}\left(y_{t} \bar{Q}_{L} H^{c} t_{R}+y_{b} \bar{Q}_{L} H b_{R}+\text { h.c. }\right)
\end{aligned}
$$

where $d_{4}$ is real as we assume a $C P$-even Higgs.

\section{A.2 Doublet Dark Matter}

The doublet DM, $\xi \sim(2,2)$, is quite involved since in this case $\xi$ has a non-zero hypercharge. However, the number of possible operators can be greatly simplified imposing $\mathrm{SO}(4)$ invariance and using suitable field redefinitions. In order to simplify the notation, we use the following $\mathrm{SO}(4)$ bi-doublets to construct the invariant operators

$$
\Phi \equiv\left(H^{c}, H\right) \quad \text { and } \quad \Phi_{\xi}=\left(\xi^{c}, \xi\right)
$$

The above $2 \times 2$ fields transform under $\mathrm{SO}(4)$ as

$$
\Phi \rightarrow L \Phi R^{\dagger} \quad \text { and } \quad \Phi_{\xi} \rightarrow L \Phi_{\xi} R^{\dagger}
$$

so $\operatorname{Tr}\left[\Phi^{\dagger} \Phi\right]$ is a $\mathrm{SO}(4)$ singlet.

For the scalar potential we can construct the following $\mathrm{SU}(2)_{L} \times \mathrm{U}(1)_{Y}$ invariant terms involving 2 powers of $\xi$

dimension 2: $\operatorname{Tr}\left[\Phi_{\xi}^{\dagger} \Phi_{\xi}\right]$;

dimension 4: $\operatorname{Tr}\left[\Phi^{\dagger} \Phi\right] \operatorname{Tr}\left[\Phi_{\xi}^{\dagger} \Phi_{\xi}\right], \operatorname{Tr}\left[\Phi^{\dagger} \Phi_{\xi}\right]^{2}$

$$
\operatorname{Tr}\left[\Phi^{\dagger} \Phi_{\xi} \sigma^{3}\right]^{2}, \operatorname{Tr}\left[\Phi^{\dagger} \Phi_{\xi}\right] \operatorname{Tr}\left[\Phi^{\dagger} \Phi_{\xi} \sigma^{3}\right] ;
$$

dimension 6: $\operatorname{Tr}\left[\Phi^{\dagger} \Phi\right]^{2} \operatorname{Tr}\left[\Phi_{\xi}^{\dagger} \Phi_{\xi}\right], \operatorname{Tr}\left[\Phi^{\dagger} \Phi\right] \operatorname{Tr}\left[\Phi^{\dagger} \Phi_{\xi}\right]^{2}$,

$$
\operatorname{Tr}\left[\Phi^{\dagger} \Phi\right] \operatorname{Tr}\left[\Phi^{\dagger} \Phi_{\xi} \sigma^{3}\right]^{2}, \operatorname{Tr}\left[\Phi^{\dagger} \Phi\right] \operatorname{Tr}\left[\Phi^{\dagger} \Phi_{\xi}\right] \operatorname{Tr}\left[\Phi^{\dagger} \Phi_{\xi} \sigma^{3}\right] .
$$

Notice that all the operators involving $\sigma^{3}$ explicitly break $\mathrm{SU}(2)_{R}$. However, they are allowed in the scalar potential since it is generated by the Yukawa and gauge couplings between the SM and the strong sector, which violate the SO(4) symmetry. All the other possible operators (including the triplet-triplet contractions) can be reduced to the ones above. Writing the above operators in terms of $\xi$ and $H$ we obtain the following scalar potential

$$
\begin{aligned}
V(H, \xi)= & \mu_{\xi}^{2}|\xi|^{2}+\lambda_{3}\left(1+\frac{\lambda_{3}^{\prime}}{F^{2}}|H|^{2}\right)|\xi|^{2}|H|^{2}+\lambda_{4}\left(1+\frac{\lambda_{4}^{\prime}}{F^{2}}|H|^{2}\right)\left|\xi^{\dagger} H\right|^{2} \\
& +\frac{\lambda_{5}}{2}\left[\left(1+\frac{\lambda_{5}^{\prime}}{F^{2}}|H|^{2}\right)\left(\xi^{\dagger} H\right)^{2}+\text { h.c. }\right] .
\end{aligned}
$$


In the doublet case there are two dimension-6 Yukawa-type operators involving $\xi$, the singlet-singlet and triplet-triplet operators

$$
\begin{aligned}
V_{\text {Yuk }}(\Phi, \xi)= & \frac{d_{4}}{F^{2}}|\xi|^{2}\left(y_{t} \bar{Q}_{L} H^{c} t_{R}+y_{b} \bar{Q}_{L} H b_{R}+\text { h.c. }\right) \\
& +\frac{d_{6}}{F^{2}} \xi^{\dagger} \vec{\sigma} \xi\left(y_{t} \bar{Q}_{L} \vec{\sigma} H^{c} t_{R}-y_{b} \bar{Q}_{L} \vec{\sigma} H b_{R}+\text { h.c. }\right) \\
& +\frac{d_{6}^{\prime}}{F^{2}}\left(y_{b} \xi^{c \dagger} \vec{\sigma} \xi \bar{Q}_{L} \vec{\sigma} H^{c} b_{R}+y_{t} \xi^{\dagger} \vec{\sigma} \xi^{c} \bar{Q}_{L} \vec{\sigma} H t_{R}+\text { h.c. }\right) .
\end{aligned}
$$

In order to avoid too many irrelevant parameters in the potential we take $d_{6}^{\prime}=d_{6}$, which we do not expect to affect any of our results.

Finally, the dimension- $6 \mathrm{SO}(4)$ invariant derivatives operators, corresponding to the effective CCWZ Lagrangian, can be constructed from the operators listed in (A.11), (A.12), (A.13)

$$
\begin{aligned}
& \operatorname{Tr}\left[\Phi^{\dagger} \Phi\right] \operatorname{Tr}\left[D_{\mu} \Phi_{\xi}^{\dagger} D^{\mu} \Phi_{\xi}\right], \quad \operatorname{Tr}\left[\Phi_{\xi}^{\dagger} \Phi_{\xi}\right] \operatorname{Tr}\left[D_{\mu} \Phi^{\dagger} D^{\mu} \Phi\right], \quad \operatorname{Tr}\left[\Phi^{\dagger} \Phi_{\xi}\right] \operatorname{Tr}\left[D_{\mu} \Phi^{\dagger} D^{\mu} \Phi_{\xi}\right], \\
& \operatorname{Tr}\left[\Phi^{\dagger} D_{\mu} \Phi_{\xi}\right]^{2}, \operatorname{Tr}\left[\Phi_{\xi}^{\dagger} D_{\mu} \Phi\right]^{2}, \operatorname{Tr}\left[\Phi^{\dagger} D_{\mu} \Phi_{\xi}\right] \operatorname{Tr}\left[\Phi_{\xi}^{\dagger} D^{\mu} \Phi\right], \quad \operatorname{Tr}\left[\Phi^{\dagger} D_{\mu} \Phi\right] \operatorname{Tr}\left[\Phi_{\xi}^{\dagger} D^{\mu} \Phi_{\xi}\right], \\
& \operatorname{Tr}\left[\Phi^{\dagger} D_{\mu} \Phi_{\xi} \Phi_{\xi}^{\dagger} D^{\mu} \Phi\right]-\operatorname{Tr}\left[\Phi^{\dagger} D_{\mu} \Phi \Phi_{\xi}^{\dagger} D^{\mu} \Phi_{\xi}\right] \text {. }
\end{aligned}
$$

The first three operators can be rewritten in terms of the others if we consider suitable field redefinitions

$$
\begin{gathered}
H \rightarrow H+a \operatorname{Tr}\left[\Phi_{\xi}^{\dagger} \Phi_{\xi}\right] H+b \operatorname{Tr}\left[\Phi_{\xi}^{\dagger} \Phi\right] \xi, \\
\xi \rightarrow \xi+a^{\prime} \operatorname{Tr}\left[\Phi^{\dagger} \Phi\right] \xi+b \operatorname{Tr}\left[\Phi^{\dagger} \Phi_{\xi}\right] H,
\end{gathered}
$$

where $a$ and $a^{\prime}$ are chosen to cancel the first two operators and $b$ is chosen to cancel the third one. Furthermore, we notice that

$$
\operatorname{Tr}\left[\Phi^{\dagger} D_{\mu} \Phi_{\xi}\right]^{2}-\operatorname{Tr}\left[\Phi_{\xi}^{\dagger} D_{\mu} \Phi\right]^{2}=\partial_{\mu} \operatorname{Tr}\left[\Phi^{\dagger} \Phi_{\xi}\right]\left(\operatorname{Tr}\left[\Phi^{\dagger} D_{\mu} \Phi_{\xi}\right]-\operatorname{Tr}\left[\Phi_{\xi}^{\dagger} D_{\mu} \Phi\right]\right) .
$$

The above operator can also be eliminated through the field redefinition

$$
H \rightarrow H+c \operatorname{Tr}\left[\Phi_{\xi}^{\dagger} \Phi\right] \xi, \quad \xi \rightarrow \xi-c \operatorname{Tr}\left[\Phi^{\dagger} \Phi_{\xi}\right] H,
$$

with the coefficient $c$ suitably chosen. Therefore, after the above field transformations we obtain only 4 independent operators, resulting in the following effective CCWZ Lagrangian $^{15}$

$$
\begin{aligned}
\mathcal{L}_{\mathrm{CCWZ}}^{(2)}= & \left(D_{\mu} \xi\right)^{\dagger} D^{\mu} \xi+\left(D_{\mu} H\right)^{\dagger} D^{\mu} H+\frac{a_{d 1}}{2 F^{2}} \operatorname{Tr}\left[\Phi^{\dagger} D_{\mu} \Phi\right] \operatorname{Tr}\left[\Phi_{\xi}^{\dagger} D^{\mu} \Phi_{\xi}\right] \\
& +\frac{a_{d 2}}{F^{2}} \operatorname{Tr}\left[\Phi^{\dagger} D_{\mu} \Phi_{\xi}\right] \operatorname{Tr}\left[\Phi_{\xi}^{\dagger} D^{\mu} \Phi\right]+\frac{a_{d 3}}{F^{2}}\left(\operatorname{Tr}\left[\Phi^{\dagger} D_{\mu} \Phi_{\xi}\right]+\operatorname{Tr}\left[\Phi_{\xi}^{\dagger} D_{\mu} \Phi\right]\right)^{2} \\
& +\frac{a_{d 4}}{F^{2}}\left(\operatorname{Tr}\left[\Phi^{\dagger} D_{\mu} \Phi_{\xi} \Phi_{\xi}^{\dagger} D^{\mu} \Phi\right]-\operatorname{Tr}\left[\Phi^{\dagger} D_{\mu} \Phi \Phi_{\xi}^{\dagger} D^{\mu} \Phi_{\xi}\right]\right) .
\end{aligned}
$$

\footnotetext{
${ }^{15}$ In (A.20) we have replaced the operator

$$
\operatorname{Tr}\left[\Phi^{\dagger} D_{\mu} \Phi_{\xi}\right]^{2}+\operatorname{Tr}\left[\Phi_{\xi}^{\dagger} D_{\mu} \Phi\right]^{2} \quad \text { by } \quad\left(\operatorname{Tr}\left[\Phi^{\dagger} D_{\mu} \Phi_{\xi}\right]+\operatorname{Tr}\left[\Phi_{\xi}^{\dagger} D_{\mu} \Phi\right]\right)^{2},
$$
}

since they only differ by the operator proportional to $a_{d 2}$. 
Finally, rewriting the operators in terms of the $H$ and $\xi$ fields and combining (A.14), (A.15) and (A.20), we obtain the effective Lagrangian for the doublet DM field

$$
\begin{aligned}
\mathcal{L}^{(2)}= & \left(D_{\mu} \xi\right)^{\dagger} D^{\mu} \xi-\mu_{\xi}^{2}|\xi|^{2}-\lambda_{3}\left(1+\frac{\lambda_{3}^{\prime}}{F^{2}}|H|^{2}\right)|\xi|^{2}|H|^{2}-\lambda_{4}\left(1+\frac{\lambda_{4}^{\prime}}{F^{2}}|H|^{2}\right)\left|\xi^{\dagger} H\right|^{2} \\
& -\frac{\lambda_{5}}{2}\left(1+\frac{\lambda_{5}^{\prime}}{F^{2}}|H|^{2}\right)\left[\left(\xi^{\dagger} H\right)^{2}+\text { h.c. }\right]+\frac{a_{d 1}}{2 F^{2}} \partial_{\mu}|H|^{2} \partial^{\mu}|\xi|^{2} \\
& +\frac{a_{d 2}}{F^{2}}\left(H^{\dagger} D_{\mu} \xi+\text { h.c. }\right)\left(\xi^{\dagger} D^{\mu} H+\text { h.c. }\right)+\frac{a_{d 3}}{F^{2}}\left[\partial_{\mu}\left(\xi^{\dagger} H+\text { h.c. }\right)\right]^{2} \\
& +\frac{a_{d 4}}{F^{2}}\left[\xi^{\dagger} \overleftrightarrow{D}_{\mu} \xi H^{\dagger} \overleftrightarrow{D}^{\mu} H+\xi^{\dagger} \overleftrightarrow{D}_{\mu} \xi^{C} H^{C \dagger} \overleftrightarrow{D}^{\mu} H-\xi^{\dagger} \vec{\sigma} \overleftrightarrow{D}{ }_{\mu} \xi H^{\dagger} \vec{\sigma} \overleftrightarrow{D}^{\mu} H+\text { h.c. }\right] \\
& -\frac{d_{4}}{F^{2}}|\xi|^{2}\left(y_{t} \bar{Q}_{L} H^{c} t_{R}+y_{b} \bar{Q}_{L} H b_{R}+\text { h.c. }\right) \\
& -\frac{d_{6}}{F^{2}}\left[\xi^{\dagger} \vec{\sigma} \xi\left(y_{t} \bar{Q}_{L} \vec{\sigma} H^{c} t_{R}-y_{b} \bar{Q}_{L} \vec{\sigma} H b_{R}\right)+y_{b} \xi^{c \dagger} \vec{\sigma} \xi \bar{Q}_{L} \vec{\sigma} H^{c} b_{R}+y_{t} \xi^{\dagger} \vec{\sigma} \xi^{c} \bar{Q}_{L} \vec{\sigma} H t_{R}+\text { h.c. }\right],
\end{aligned}
$$

where $d_{4}$ and $d_{6}$ are real as we assume that the Higgs doublet is $C P$-even. We have verified that the imaginary parts of $\lambda_{5}$ and $\lambda_{5}^{\prime}$ have almost no impact on the allowed parameter space. Therefore, for simplicity, we take these complex parameters as real.

\section{A.3 Real representations}

Here we discuss the case where $\xi$ is a real multiplet, which corresponds to the $\mathrm{SO}(4)$ representations $(n, 1)$. The possible operators involving a real $\xi$ multiplet are greatly reduced due to the identities

$$
\xi^{\dagger} \overleftrightarrow{D^{\mu}} \xi=\left(D_{\mu} \xi\right)^{\dagger} \vec{\Gamma} \xi+\text { h.c. }=\xi^{\dagger} \vec{\Gamma} \xi=\left(D_{\mu} \xi\right)^{\dagger} \vec{\Gamma} D^{\mu} \xi=0 .
$$

Furthermore, since $\xi$ is a singlet under $\mathrm{SU}(2)_{R}$, the $\mathrm{SO}(4)$ invariance implies that $\xi$ can only couple to $\mathrm{SU}(2)_{R}$ singlets. The only possible operators contributing to the scalar potential with two powers of $\xi$ are

dimension 2: $\operatorname{Tr}\left[\Phi_{\xi}^{\dagger} \Phi_{\xi}\right]$;

dimension 4: $\operatorname{Tr}\left[\Phi^{\dagger} \Phi\right] ; \operatorname{Tr}\left[\Phi_{\xi}^{\dagger} \Phi_{\xi}\right]$

dimension 6: $\operatorname{Tr}\left[\Phi^{\dagger} \Phi\right]^{2} \operatorname{Tr}\left[\Phi_{\xi}^{\dagger} \Phi_{\xi}\right], \operatorname{Tr}\left[\Phi^{\dagger} \sigma^{i} \Phi \sigma^{3}\right] \operatorname{Tr}\left[\Phi^{\dagger} \sigma^{j} \Phi \sigma^{3}\right] \xi^{\dagger}\left\{\Gamma^{i}, \Gamma^{j}\right\} \xi$,

$$
\operatorname{Tr}\left[\Phi^{\dagger} \sigma^{i} \Phi \sigma^{+}\right] \operatorname{Tr}\left[\Phi^{\dagger} \sigma^{j} \Phi \sigma^{-}\right] \xi^{\dagger}\left\{\Gamma^{i}, \Gamma^{j}\right\} \xi
$$

where $\sigma^{ \pm}=\sigma^{1} \pm i \sigma^{2}$. The dimension-6 derivative operators invariant under $\mathrm{SO}(4)$, which parametrize the effective CCWZ Lagrangian, are given by

$$
\begin{array}{lll}
\operatorname{Tr}\left[\Phi^{\dagger} \Phi\right]\left(D_{\mu} \xi\right)^{\dagger} D^{\mu} \xi, & & |\xi|^{2} \operatorname{Tr}\left[D^{\mu} \Phi^{\dagger} D_{\mu} \Phi\right] \\
\operatorname{Tr}\left[\Phi^{\dagger} D_{\mu} \Phi\right] \partial^{\mu}|\xi|^{2} & \text { and } & \operatorname{Tr}\left[\Phi^{\dagger} \vec{\sigma} D_{\mu} \Phi\right] \xi^{\dagger} \vec{\Gamma} \xi
\end{array}
$$

As before, the first two operators can be eliminated through the field rescalings:

$$
H \rightarrow H+a|\xi|^{2} H \text { and } \xi \rightarrow \xi+a^{\prime}|H|^{2} \xi
$$


Therefore, writing the above operators in terms of the $H$ and $\xi$ fields we obtain

$$
\begin{aligned}
\mathcal{L}^{(2)}= & \left(D_{\mu} \xi\right)^{\dagger} D^{\mu} \xi-\mu_{\xi}^{2}|\xi|^{2}-\lambda_{3}\left(1+\frac{\lambda_{3}^{\prime}}{F^{2}}|H|^{2}\right)|\xi|^{2}|H|^{2} \\
& -\frac{\lambda_{4}}{F^{2}} \xi^{\dagger}\left\{\Gamma^{i}, \Gamma^{j}\right\} \xi H^{\dagger} \sigma^{i} H H^{\dagger} \sigma^{j} H-\frac{\lambda_{5}}{F^{2}} \xi^{\dagger}\left\{\Gamma^{i}, \Gamma^{j}\right\} \xi H^{c \dagger} \sigma^{i} H H^{\dagger} \sigma^{j} H^{c} \\
& +\frac{a_{d 1}}{2 F^{2}} \partial_{\mu}|\xi|^{2} \partial^{\mu}|H|^{2}-\frac{a_{d 4}}{F^{2}} \xi^{\dagger} \vec{\Gamma} \overleftrightarrow{D}^{\mu} \xi H^{\dagger} \vec{\sigma} \overleftrightarrow{D}_{\mu} H \\
& -\frac{d_{4}}{F^{2}}|\xi|^{2}\left(y_{t} \bar{Q}_{L} H^{c} t_{R}+y_{b} \bar{Q}_{L} H b_{R}+\text { h.c. }\right),
\end{aligned}
$$

where once again we take $d_{4}$ to be real as we assume a $C P$-even Higgs.

Open Access. This article is distributed under the terms of the Creative Commons Attribution License (CC-BY 4.0), which permits any use, distribution and reproduction in any medium, provided the original author(s) and source are credited.

\section{References}

[1] CMS collaboration, Observation of a new boson at a mass of $125 \mathrm{GeV}$ with the CMS experiment at the LHC, Phys. Lett. B 716 (2012) 30 [arXiv:1207.7235] [INSPIRE].

[2] ATLAS collaboration, Observation of a new particle in the search for the Standard Model Higgs boson with the ATLAS detector at the LHC, Phys. Lett. B 716 (2012) 1 [arXiv: 1207.7214] [INSPIRE].

[3] F. Englert and R. Brout, Broken Symmetry and the Mass of Gauge Vector Mesons, Phys. Rev. Lett. 13 (1964) 321 [INSPIRE].

[4] P.W. Higgs, Broken symmetries, massless particles and gauge fields, Phys. Lett. 12 (1964) 132 [INSPIRE].

[5] P.W. Higgs, Broken Symmetries and the Masses of Gauge Bosons, Phys. Rev. Lett. 13 (1964) 508 [INSPIRE].

[6] H. Baer and X. Tata, Weak scale supersymmetry: From superfields to scattering events, Cambridge University Press, Cambridge, U.K. (2006).

[7] M. Drees, R. Godbole and P. Roy, Theory and phenomenology of sparticles: An account of four-dimensional $N=1$ supersymmetry in high energy physics, World Scientific, (2004) [INSPIRE].

[8] S.P. Martin, A supersymmetry primer, Adv. Ser. Direct. High Energy Phys. 21 (2010) 1 [hep-ph/9709356] [INSPIRE].

[9] D.B. Kaplan and H. Georgi, SU(2) $\times$ U(1) Breaking by Vacuum Misalignment, Phys. Lett. B 136 (1984) 183 [INSPIRE].

[10] D.B. Kaplan, H. Georgi and S. Dimopoulos, Composite Higgs Scalars, Phys. Lett. B 136 (1984) 187 [INSPIRE].

[11] T. Banks, Constraints on $\mathrm{SU}(2) \times \mathrm{U}(1)$ breaking by vacuum misalignment, Nucl. Phys. B 243 (1984) 125 [INSPIRE].

[12] H. Georgi, D.B. Kaplan and P. Galison, Calculation of the Composite Higgs Mass, Phys. Lett. B 143 (1984) 152 [inSPIRE]. 
[13] H. Georgi and D.B. Kaplan, Composite Higgs and Custodial SU(2), Phys. Lett. B 145 (1984) 216 [INSPIRE].

[14] M.J. Dugan, H. Georgi and D.B. Kaplan, Anatomy of a Composite Higgs Model, Nucl. Phys. B 254 (1985) 299 [INSPIRE].

[15] K. Agashe, R. Contino and A. Pomarol, The Minimal composite Higgs model, Nucl. Phys. B 719 (2005) 165 [hep-ph/0412089] [INSPIRE].

[16] M. Frigerio, A. Pomarol, F. Riva and A. Urbano, Composite Scalar Dark Matter, JHEP 07 (2012) 015 [arXiv: 1204.2808] [INSPIRE].

[17] D. Marzocca and A. Urbano, Composite Dark Matter and LHC Interplay, JHEP 07 (2014) 107 [arXiv: 1404.7419] [INSPIRE].

[18] M. Chala, $h \rightarrow \gamma \gamma$ excess and Dark Matter from Composite Higgs Models, JHEP 01 (2013) 122 [arXiv:1210.6208] [INSPIRE].

[19] LUX collaboration, D.S. Akerib et al., First results from the LUX dark matter experiment at the Sanford Underground Research Facility, Phys. Rev. Lett. 112 (2014) 091303 [arXiv: 1310.8214] [INSPIRE].

[20] FERMi-LAT collaboration, M. Ackermann et al., Dark matter constraints from observations of 25 Milky Way satellite galaxies with the Fermi Large Area Telescope, Phys. Rev. D 89 (2014) 042001 [arXiv: 1310.0828] [INSPIRE].

[21] B. Anderson, A search for dark matter annihilation in Dwarf Spheroidal Galaxies with Pass 8 Data, in proceedings of The Fifth International Fermi Symposium, Nagoya, Japan, 20-24 October 2014.

[22] T. Bringmann and C. Weniger, Gamma Ray Signals from Dark Matter: Concepts, Status and Prospects, Phys. Dark Univ. 1 (2012) 194 [arXiv: 1208.5481] [inSPIRE].

[23] N. Zhou, Z. Khechadoorian, D. Whiteson and T.M.P. Tait, Bounds on Invisible Higgs boson Decays from $t \bar{t} H$ Production, Phys. Rev. Lett. 113 (2014) 151801 [arXiv:1408.0011] [INSPIRE].

[24] ATLAS collaboration, Search for Invisible Decays of a Higgs Boson Produced in Association with a Z Boson in ATLAS, Phys. Rev. Lett. 112 (2014) 201802 [arXiv:1402.3244] [INSPIRE].

[25] CMS collaboration, Search for invisible decays of Higgs bosons in the vector boson fusion and associated ZH production modes, Eur. Phys. J. C 74 (2014) 2980 [arXiv:1404.1344] [INSPIRE].

[26] Planck collaboration, P.A.R. Ade et al., Planck 2013 results. XVI. Cosmological parameters, Astron. Astrophys. 571 (2014) A16 [arXiv:1303.5076] [INSPIRE].

[27] L. Lopez-Honorez, O. Mena, S. Palomares-Ruiz and A.C. Vincent, Constraints on dark matter annihilation from CMB observationsbefore Planck, JCAP 07 (2013) 046 [arXiv: 1303.5094] [INSPIRE].

[28] M.S. Madhavacheril, N. Sehgal and T.R. Slatyer, Current Dark Matter Annihilation Constraints from CMB and Low-Redshift Data, Phys. Rev. D 89 (2014) 103508 [arXiv: 1310.3815] [INSPIRE].

[29] S. Galli, Preliminary Planck results on DM annihilation, talk given at Planck 2014 - The microwave sky in temperature and polarization, Ferrara, Italy, 1-5 December 2014. 
[30] J. Mrazek et al., The Other Natural Two Higgs Doublet Model, Nucl. Phys. B 853 (2011) 1 [arXiv: 1105.5403] [INSPIRE].

[31] B. Gripaios, A. Pomarol, F. Riva and J. Serra, Beyond the Minimal Composite Higgs Model, JHEP 04 (2009) 070 [arXiv:0902.1483] [INSPIRE].

[32] Y. Kikuta, Y. Okada and Y. Yamamoto, Structure of dimension-six derivative interactions in pseudo Nambu-Goldstone N Higgs doublet models, Phys. Rev. D 85 (2012) 075021 [arXiv:1111.2120] [INSPIRE].

[33] R. Alonso, I. Brivio, B. Gavela, L. Merlo and S. Rigolin, Sigma Decomposition, JHEP 12 (2014) 034 [arXiv: 1409.1589] [INSPIRE].

[34] A. Carmona and F. Goertz, A naturally light Higgs without light Top Partners, JHEP 05 (2015) 002 [arXiv: 1410.8555] [INSPIRE].

[35] T. Alanne, H. Gertov, F. Sannino and K. Tuominen, Elementary Goldstone Higgs boson and dark matter, Phys. Rev. D 91 (2015) 095021 [arXiv:1411.6132] [InSPIRE].

[36] T.A. Ryttov and F. Sannino, Ultra Minimal Technicolor and its Dark Matter TIMP, Phys. Rev. D 78 (2008) 115010 [arXiv:0809.0713] [INSPIRE].

[37] S.R. Coleman, J. Wess and B. Zumino, Structure of phenomenological Lagrangians. 1., Phys. Rev. 177 (1969) 2239 [INSPIRE].

[38] C.G. Callan Jr., S.R. Coleman, J. Wess and B. Zumino, Structure of phenomenological Lagrangians. 2, Phys. Rev. 177 (1969) 2247 [InSPIRE].

[39] G. Panico, M. Redi, A. Tesi and A. Wulzer, On the Tuning and the Mass of the Composite Higgs, JHEP 03 (2013) 051 [arXiv:1210.7114] [InSPIRE].

[40] M. Baak et al., The Electroweak Fit of the Standard Model after the Discovery of a New Boson at the LHC, Eur. Phys. J. C 72 (2012) 2205 [arXiv: 1209.2716] [InSPIRE].

[41] A. Falkowski, F. Riva and A. Urbano, Higgs at last, JHEP 11 (2013) 111 [arXiv:1303.1812] [INSPIRE].

[42] T. Hambye, F.-S. Ling, L. Lopez Honorez and J. Rocher, Scalar Multiplet Dark Matter, JHEP 07 (2009) 090 [arXiv: 0903.4010] [INSPIRE].

[43] X.-1. Chen and M. Kamionkowski, Three body annihilation of neutralinos below two-body thresholds, JHEP 07 (1998) 001 [hep-ph/9805383] [INSPIRE].

[44] Y. Hosotani, P. Ko and M. Tanaka, Stable Higgs Bosons as Cold Dark Matter, Phys. Lett. B 680 (2009) 179 [arXiv:0908.0212] [INSPIRE].

[45] C.E. Yaguna, Large contributions to dark matter annihilation from three-body final states, Phys. Rev. D 81 (2010) 075024 [arXiv:1003.2730] [INSPIRE].

[46] L. Lopez Honorez and C.E. Yaguna, The inert doublet model of dark matter revisited, JHEP 09 (2010) 046 [arXiv: 1003.3125] [INSPIRE].

[47] G. Bélanger, F. Boudjema, A. Pukhov and A. Semenov, MicrOMEGAs_3: A program for calculating dark matter observables, Comput. Phys. Commun. 185 (2014) 960 [arXiv: 1305.0237] [INSPIRE].

[48] G.F. Giudice, C. Grojean, A. Pomarol and R. Rattazzi, The Strongly-Interacting Light Higgs, JHEP 06 (2007) 045 [hep-ph/0703164] [INSPIRE]. 
[49] T. Corbett, O.J.P. Eboli, J. Gonzalez-Fraile and M.C. Gonzalez-Garcia, Robust Determination of the Higgs Couplings: Power to the Data, Phys. Rev. D 87 (2013) 015022 [arXiv: 1211.4580] [INSPIRE].

[50] R. Contino, M. Ghezzi, C. Grojean, M. Muhlleitner and M. Spira, Effective Lagrangian for a light Higgs-like scalar, JHEP 07 (2013) 035 [arXiv:1303.3876] [INSPIRE].

[51] J. Elias-Miró, J.R. Espinosa, E. Masso and A. Pomarol, Renormalization of dimension-six operators relevant for the Higgs decays $h \rightarrow \gamma \gamma, \gamma Z$, JHEP 08 (2013) 033 [arXiv:1302.5661] [INSPIRE].

[52] J. Elias-Miro, J.R. Espinosa, E. Masso and A. Pomarol, Higgs windows to new physics through $D=6$ operators: constraints and one-loop anomalous dimensions, JHEP 11 (2013) 066 [arXiv:1308.1879] [INSPIRE].

[53] R. Alonso, M.B. Gavela, L. Merlo, S. Rigolin and J. Yepes, The Effective Chiral Lagrangian for a Light Dynamical "Higgs Particle", Phys. Lett. B 722 (2013) 330 [arXiv:1212.3305] [INSPIRE].

[54] J. Ellis, V. Sanz and T. You, The Effective Standard Model after LHC Run I, JHEP 03 (2015) 157 [arXiv:1410.7703] [INSPIRE].

[55] G. Jungman, M. Kamionkowski and K. Griest, Supersymmetric dark matter, Phys. Rept. 267 (1996) 195 [hep-ph/9506380] [INSPIRE].

[56] J. Mrazek et al., The Other Natural Two Higgs Doublet Model, Nucl. Phys. B 853 (2011) 1 [arXiv: 1105.5403] [INSPIRE].

[57] K. Griest and M. Kamionkowski, Unitarity Limits on the Mass and Radius of Dark Matter Particles, Phys. Rev. Lett. 64 (1990) 615 [InSPIRE].

[58] T. Corbett, O. Éboli and M. Gonzalez-Garcia, Unitarity Constraints on Dimension-Six Operators, Phys. Rev. D 91 (2015) 035014 [arXiv:1411.5026] [InSPIRE].

[59] WMAP collaboration, G. Hinshaw et al., Nine-Year Wilkinson Microwave Anisotropy Probe (WMAP) Observations: Cosmological Parameter Results, Astrophys. J. Suppl. 208 (2013) 19 [arXiv: 1212.5226] [INSPIRE].

[60] G. Bélanger, F. Boudjema, A. Pukhov and A. Semenov, Dark matter direct detection rate in a generic model with MicrOMEGAs 2.2, Comput. Phys. Commun. 180 (2009) 747 [arXiv:0803.2360] [INSPIRE].

[61] A. Alloul, N.D. Christensen, C. Degrande, C. Duhr and B. Fuks, FeynRules 2.0 - A complete toolbox for tree-level phenomenology, Comput. Phys. Commun. 185 (2014) 2250 [arXiv:1310.1921] [INSPIRE].

[62] G. Bélanger, F. Boudjema, A. Pukhov and A. Semenov, MicrOMEGAs4.1: two dark matter candidates, Comput. Phys. Commun. 192 (2015) 322 [arXiv:1407.6129] [InSPIRE].

[63] M. Cirelli, N. Fornengo and A. Strumia, Minimal dark matter, Nucl. Phys. B 753 (2006) 178 [hep-ph/0512090] [INSPIRE].

[64] M. Klasen, C.E. Yaguna and J.D. Ruiz-Alvarez, Electroweak corrections to the direct detection cross section of inert Higgs dark matter, Phys. Rev. D 87 (2013) 075025 [arXiv: 1302.1657] [INSPIRE].

[65] T. Abe and R. Sato, Quantum corrections to the spin-independent cross section of the inert doublet dark matter, JHEP 03 (2015) 109 [arXiv: 1501.04161] [INSPIRE]. 
[66] WMAP collaboration, E. Komatsu et al., Five-Year Wilkinson Microwave Anisotropy Probe (WMAP) Observations: Cosmological Interpretation, Astrophys. J. Suppl. 180 (2009) 330 [arXiv: 0803.0547] [INSPIRE].

[67] PAMELA collaboration, O. Adriani et al., PAMELA Measurements of Cosmic-ray Proton and Helium Spectra, Science 332 (2011) 69 [arXiv:1103.4055] [INSPIRE].

[68] O. Adriani et al., Measurement of the flux of primary cosmic ray antiprotons with energies of 60-MeV to 350-GeV in the PAMELA experiment, JETP Lett. 96 (2013) 621 [INSPIRE].

[69] C. Evoli, I. Cholis, D. Grasso, L. Maccione and P. Ullio, Antiprotons from dark matter annihilation in the Galaxy: astrophysical uncertainties, Phys. Rev. D 85 (2012) 123511 [arXiv:1108.0664] [INSPIRE].

[70] M. Cirelli, D. Gaggero, G. Giesen, M. Taoso and A. Urbano, Antiproton constraints on the GeV gamma-ray excess: a comprehensive analysis, JCAP 12 (2014) 045 [arXiv:1407.2173] [INSPIRE].

[71] S. Kraml et al., SModelS: a tool for interpreting simplified-model results from the LHC and its application to supersymmetry, Eur. Phys. J. C 74 (2014) 2868 [arXiv:1312.4175] [INSPIRE].

[72] R. Barbieri, L.J. Hall and V.S. Rychkov, Improved naturalness with a heavy Higgs: An alternative road to LHC physics, Phys. Rev. D 74 (2006) 015007 [hep-ph/0603188] [INSPIRE].

[73] L. Lopez Honorez, E. Nezri, J.F. Oliver and M.H.G. Tytgat, The Inert Doublet Model: An Archetype for Dark Matter, JCAP 02 (2007) 028 [hep-ph/0612275] [INSPIRE].

[74] I.F. Ginzburg, K.A. Kanishev, M. Krawczyk and D. Sokolowska, Evolution of Universe to the present inert phase, Phys. Rev. D 82 (2010) 123533 [arXiv: 1009.4593] [INSPIRE].

[75] E. Lundstrom, M. Gustafsson and J. Edsjo, The Inert Doublet Model and LEP II Limits, Phys. Rev. D 79 (2009) 035013 [arXiv:0810.3924] [InSPIRE].

[76] Q.-H. Cao, E. Ma and G. Rajasekaran, Observing the Dark Scalar Doublet and its Impact on the Standard-Model Higgs Boson at Colliders, Phys. Rev. D 76 (2007) 095011 [arXiv:0708.2939] [INSPIRE].

[77] A. Pierce and J. Thaler, Natural Dark Matter from an Unnatural Higgs Boson and New Colored Particles at the TeV Scale, JHEP 08 (2007) 026 [hep-ph/0703056] [INSPIRE].

[78] M. Gustafsson, The Inert Doublet Model and Its Phenomenology, PoS (CHARGED 2010) 030 [arXiv:1106.1719] [INSPIRE].

[79] L. Lopez Honorez and C.E. Yaguna, A new viable region of the inert doublet model, JCAP 01 (2011) 002 [arXiv: 1011.1411] [inSPIRE].

[80] M. Cirelli, A. Strumia and M. Tamburini, Cosmology and Astrophysics of Minimal Dark Matter, Nucl. Phys. B 787 (2007) 152 [arXiv:0706.4071] [INSPIRE].

[81] A. Sommerfeld, Diffraction and Retardation of Electrons, Annalen Phys. 403 (1931) 257.

[82] K. Agashe, A. Delgado, M.J. May and R. Sundrum, RS1, custodial isospin and precision tests, JHEP 08 (2003) 050 [hep-ph/0308036] [INSPIRE].

[83] G. Burdman, N. Fonseca and L. de Lima, Full-hierarchy Quiver Theories of Electroweak Symmetry Breaking and Fermion Masses, JHEP 01 (2013) 094 [arXiv:1210.5568] [INSPIRE]. 